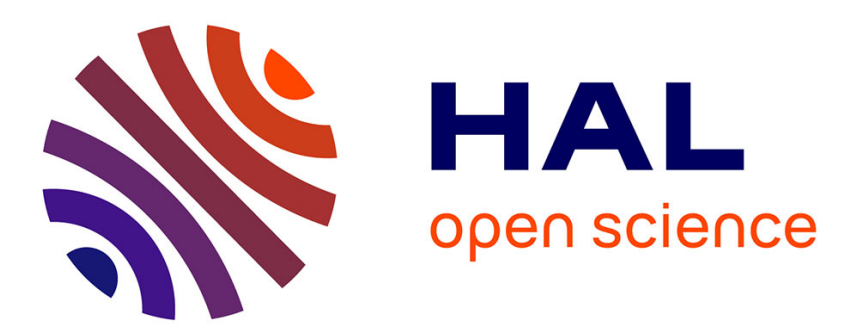

\title{
A polynomial chaos approach to narrow band modeling of radiative heat transfer in non-uniform gaseous media
}

Frédéric André

\section{To cite this version:}

Frédéric André. A polynomial chaos approach to narrow band modeling of radiative heat transfer in non-uniform gaseous media. Journal of Quantitative Spectroscopy and Radiative Transfer, 2016, 175, pp.17-29. 10.1016/j.jqsrt.2016.01.037 . hal-02064933

\section{HAL Id: hal-02064933 \\ https://hal.science/hal-02064933}

Submitted on 23 Apr 2019

HAL is a multi-disciplinary open access archive for the deposit and dissemination of scientific research documents, whether they are published or not. The documents may come from teaching and research institutions in France or abroad, or from public or private research centers.
L'archive ouverte pluridisciplinaire HAL, est destinée au dépôt et à la diffusion de documents scientifiques de niveau recherche, publiés ou non, émanant des établissements d'enseignement et de recherche français ou étrangers, des laboratoires publics ou privés. 


\section{A POLYNOMIAL CHAOS APPROACH TO NARROW BAND MODELLING OF RADIATIVE HEAT TRANSFER IN NON-UNIFORM GASEOUS MEDIA}

Frédéric André

Université de Lyon, CNRS

INSA-Lyon, CETHIL, UMR5008, F-69621, Villeurbanne, France

Université Lyon 1, CETHIL, UMR5008, F-69622, France

*Corresponding author, e-mail: frederic.andre@insa-lyon.fr, tel: +33 (0)4 724388 16, fax: +33 (0)4 72438811 


\begin{abstract}
An accurate treatment of non-uniformities is required in many applications involving radiative heat transfer in gaseous media. Usual techniques to handle path non-uniformities rely on simplifying assumptions, such as scaling or correlation of gas spectra. Those approximations are usually accurate but may also fail to provide accurate results, especially when large temperature gradients are considered. The objective of the present work is to show that this problem can be treated rigorously. The proposed method can be applied to any arbitrary narrow band model. It is based on some results from Polynomial Chaos' framework and copulas theory. Although the mathematical derivation may appear sophisticated, applying the method is straightforward. It is shown that adding only one coefficient to any uniform narrow band model (for a simple case involving a non-uniform column discretized into two uniform sub-paths) allows to achieve almost LBL accuracy for radiative heat transfer calculations. The technique is described and applied to some "severe" test cases from the literature
\end{abstract}

KEYWORDS: gas radiation, polynomial chaos, copulas, Cutteridge-Devyatov polynomial chaos, $\ell$-distribution, water vapor. 


\section{INTRODUCTION}

Many approximate models are available in the literature to treat radiative heat transfer in nonuniform gaseous media. The so-called Line-By-Line technique accounts for each line of the absorbing species at high spectral resolution. It is widely recognized as the most accurate approach. Nevertheless, it is computationally too expensive to be applied in many practical situations involving multi-dimensional geometries, such as encountered frequently in many industrial and academic applications. Accordingly, and despite the large increase in terms of computational resources observed in the recent years, its use still remains restricted to the definition of reference calculations against which more computationally efficient approaches can be assessed.

Over narrow bands, there are two main categories of approximate models. Statistical Narrow Band (SNB) approaches [1,2] are based on a simplified representation of the absorption spectrum: spectral lines are assumed to have their centers randomly located inside the interval and their individual contributions to the total absorption are assumed to be uncorrelated. Several SNB models are available in the literature. They are based on different mathematical forms for the distributions of the linestrengths. The formulation proposed by Malkmus [3] was shown in Ref. [4] to be the most reliable for applications in combustion. The other class of models, usually referred to as $k$-distribution approaches, was originally proposed by Ambartzumian [5]. It mainly consists of a reordering of the wavenumber axis with respect to the values taken by the absorption coefficient. Over this reordered axis, the absorption coefficient varies smoothly. Accordingly, this process enables to transform any integral over wavenumbers - over which the absorption coefficient varies very quickly - into an integral over a reordered wavenumber scale for which computationally efficient integration schemes based on low order numerical quadratures can be used.

Although the two previous categories of models provide very accurate estimates of the radiative properties of gases over uniform paths, they require additional assumptions for applications in non-uniform media. The most common are the Curtis-Godson (CG) approximation [6] in the frame of SNB models and the Correlated- $k(\mathrm{C}-k)$ assumption [7] in the case of $k$-distributions. More details about those two approximate treatments of nonuniformities can be found, for instance, in Refs. [2,6-8]. These methods usually provide reliable estimates of the radiative properties of gases over non-uniform paths. However, they may fail to yield accurate results as soon as large temperature gradients are encountered along the path. This is physically due to the appearance in the absorption spectrum of so-called hot lines at high temperature that breaks the ideal behavior of the spectrum assumed in the CG/C$k$ approximations. The treatment of non-uniformities is usually the main source of errors of SNB-CG/C- $k$ models.

The main objective of the present work is to show how it is possible to handle path nonuniformities without any recourse to simplifying assumptions (such as scaling or correlation of absorption spectra). The mathematical derivation is funded on results from generalized Polynomial Chaos' (gPC, [9]) framework. A similar result was provided in the frame of $k$ distribution models in Ref. [10] but based on a different approach. Here, the treatment can be applied to any arbitrary narrow band model.

As the aim of this work is primarily to introduce new concepts to treat spectral correlations, only "simple" cases for which the non-uniform paths can be modeled as the juxtaposition of two uniform gaseous columns will be considered. However, 1/ those cases are known as severe, which means that most usual approaches (SNB-CG, C- $k$ ) are known to be 
inappropriate. Some tests for which usual approximate treatments of non-uniformities are known to work are also investigated to illustrate the generality of the present approach; $2 /$ the extension of the technique to more than two uniform paths is discussed at the end of the paper. Some connections with copula's theory, which is an equivalent way to treat the spectral correlation issue, are also emphasized.

The paper is structured as follows. In the second section, we derive an explicit formula to estimate the absorptivity of a non-uniform path (here made of two uniform sub-paths) when only the absorptivities of each of the sub-paths are known. This is to our knowledge the first time that this kind of exact calculation is reported in the literature. This formula involves a series expansion in terms of orthogonal polynomials. The coefficients in the series, reformulated in terms of Cutteridge-Devyatov polynomials, can be calculated directly from LBL data. A method to estimate those quantities is proposed in Section 3. The same section provides some comparisons of the present technique with reference LBL calculations for various orders of the series expansion. It is shown to be both accurate and simple to apply when the non-uniform path can be represented as the juxtaposition of two uniform sub-paths. Several possible techniques to extend the method to more than two uniform sub-paths are briefly presented at the end of the paper.

\section{PRINCIPLE OF THE METHOD}

\subsection{Polynomial chaos}

Let $f: \mathbb{R}^{n} \rightarrow \mathbb{R}$ be a deterministic function of $n$ variables. If its inputs are random variables with prescribed density probability functions, the output of function $f$ is also a Random Variable (RV). Such a RV is said to be second order if its expectancy and variance (or equivalently its first and second moments) are finite. The space of second order random variables is a Hilbert space over which a scalar product can be defined [9].

The idea behind $\mathrm{gPC}$ is to represent any such RV as a product of one dimensional sequences of polynomials orthogonal in the weighted $L^{2}$ sense. This means that over some fixed interval $I$ in the real line and for some given weight function $w(x)$ (that corresponds here to the density probability function associated with each of the input RVs), any couple of one dimensional polynomials $P_{i}$ and $P_{j}$ inside the same sequence satisfies (here $\int_{I}\left[P_{i}(x)\right]^{2} w(x) d x=1$ is assumed):

$$
\int_{I} P_{i}(x) P_{j}(x) w(x) d x=\delta_{i j}
$$

where $\delta_{i j}$ is the Kronecker Delta symbol which is 1 if $i=j$ and 0 if $i \neq j$. More details about orthogonal polynomials can be found in Refs. [11,12].

Generalized Polynomial Chaos [9] (gPC) is an extension of the concept of Polynomial Chaos introduced by Wiener in Ref. [13] in which only Hermite polynomials were considered. In gPC, a wide range of possible polynomial basis can be used (such as the shifted Legendre polynomials which are orthogonal in the interval $[0,1]$ with unit weights in the case of uniformly distributed inputs - those polynomials will be considered later in this work). 
It was shown by Cameron and Martin [14] that if the output RV $f$ is second order then its representation in terms of orthogonal polynomials converges to $f$ in the (mean square) $L^{2}$ sense. gPC is widely used in engineering problems as a tool for uncertainty quantification [15], to build metamodels [16], etc. In this work, this approach is used to derive an exact treatment of non-uniformities. This is the aim of the next section.

\subsection{Mathematical formulation}

Let us consider a narrow spectral interval $\Delta \eta$ that: $H 1 /$ is narrow enough to assume that the Planck function remains constant, $\mathrm{H} 2 /$ do not to contain any transparency region of the gas at any temperature. In order to simplify the derivation, a mixture of a single absorbing molecule $\left(\mathrm{CO}_{2}, \mathrm{CO}\right.$ or $\left.\mathrm{H}_{2} \mathrm{O}\right)$ with a non-absorbing gas (nitrogen for instance) is considered. Our problem can be stated as follows.

We consider a non-uniform path divided into two uniform sub-paths. The thermophysical state in the first sub-path (index 1) is represented by the state vector $\underline{\phi_{1}}$ that encompasses all the quantities required to characterize the gas over path 1 (its temperature, total pressure and molar fractions in absorbing species). The corresponding spectral absorption coefficient will be from now on written $\kappa_{\eta, 1}=\kappa_{\eta}\left(\underline{\phi}_{1}\right)$. The second sub-path (index 2) is, in a similar way, associated with the absorption coefficient $\kappa_{\eta, 2}=\kappa_{\eta}\left(\underline{\phi}_{2}\right)$, where $\underline{\phi}_{2}$ is the state vector that characterizes this second sub-path.

We define, for each uniform element, the narrow band averaged absorptivity of a path of length $L_{i}, i=1,2$ as:

$$
\alpha_{i}^{\Delta \eta}\left(L_{i}\right)=\frac{1}{\Delta \eta} \int_{\Delta \eta}\left[1-\exp \left(-\kappa_{\eta, i} L_{i}\right)\right] d \eta
$$

as well as their derivatives with respect to the lengths of the gas paths:

$$
\partial \alpha_{i}^{\Delta \eta}\left(L_{i}\right) / \partial L_{i}=\frac{1}{\Delta \eta} \int_{\Delta \eta} \kappa_{\eta, i} \exp \left(-\kappa_{\eta, i} L_{i}\right) d \eta
$$

Those absorptivities have the following properties, as soon as assumption $H 2$ is correct: $P 1 /$ they take values between 0 (when $L_{i}=0$ ) and 1 (when $L_{i}=+\infty$ ), $P 2 /$ they are continuous and strictly increasing (see Eq. (3)). Mathematically, they are thus distribution functions [17]. They will be referred to as $\ell$-distributions in the following.

The narrow band averaged absorptivity of the non-uniform path $L_{1}+L_{2}$ is defined as [8] (see Eq. (19.71) p. 605):

$$
\alpha_{12}^{\Delta \eta}\left(L_{1}, L_{2}\right)=\frac{1}{\Delta \eta} \int_{\Delta \eta}\left[1-\exp \left(-\kappa_{\eta, 1} L_{1}-\kappa_{\eta, 2} L_{2}\right)\right] d \eta
$$

It may be equivalently rewritten as: 


$$
\alpha_{12}^{\Delta \eta}\left(L_{1}, L_{2}\right)=\alpha_{1}^{\Delta \eta}\left(L_{1}\right)+\alpha_{2}^{\Delta \eta}\left(L_{2}\right)-\Gamma_{12}^{\Delta \eta}\left(L_{1}, L_{2}\right)
$$

where:

$$
\Gamma_{12}^{\Delta \eta}\left(L_{1}, L_{2}\right)=\frac{1}{\Delta \eta} \int_{\Delta \eta}\left[1-\exp \left(-\kappa_{\eta, 1} L_{1}\right)\right]\left[1-\exp \left(-\kappa_{\eta, 2} L_{2}\right)\right] d \eta
$$

Following Eq. (5), $\Gamma_{12}^{\Delta \eta}\left(L_{1}, L_{2}\right)$ gathers all the information about the spectral correlations. Our objective is to estimate this function.

For this purpose, let us introduce two uncorrelated random variables $\xi_{1}$ and $\xi_{2}$ uniformly distributed inside the interval $[0,1]$. Following properties $P 1-2$, for any value of $\xi_{i} \in[0,1], i=1,2$ we can define a unique length $l_{i}\left(\xi_{i}\right), i=1,2$ as a solution $\left(l_{i}\left(\xi_{i}\right)=+\infty\right.$ is allowed) of the following implicit equation:

$$
\alpha_{i}^{\Delta \eta}\left[l_{i}\left(\xi_{i}\right)\right]=\frac{1}{\Delta \eta} \int_{\Delta \eta}\left[1-\exp \left(-\kappa_{\eta, i} l_{i}\left(\xi_{i}\right)\right)\right] d \eta=\xi_{i}
$$

The previous relationship produces two new random variables, $l_{i}\left(\xi_{i}\right), i=1,2$. Each variable $l_{i}\left(\xi_{i}\right), i=1,2$ is distributed according to its own $\ell$-distribution function $\alpha_{i}^{\Delta \eta}$. This follows directly from their definition Eq. (7) and by application of the so-called inverse transformation theorem [17]. This theorem is widely used in Monte Carlo methods.

Random variables $l_{i}\left(\xi_{i}\right), i=1,2$ can be put inside Eq. (6) to construct a bivariate random function of variables $\xi_{1}$ and $\xi_{2}$ as:

$$
C\left(\xi_{1}, \xi_{2}\right)=\Gamma_{12}^{\Delta \eta}\left[l_{1}\left(\xi_{1}\right), l_{2}\left(\xi_{2}\right)\right]=\frac{1}{\Delta \eta} \int_{\Delta \eta}\left[1-\exp \left(-\kappa_{\eta, 1} l_{1}\left(\xi_{1}\right)\right)\right]\left[1-\exp \left(-\kappa_{\eta, 2} l_{2}\left(\xi_{2}\right)\right)\right] d \eta
$$

Now, we can notice that for any $\left(\xi_{1}, \xi_{2}\right) \in[0,1]^{2}$, we have: $0 \leq C\left(\xi_{1}, \xi_{2}\right) \leq 1$. This shows that:

$$
\left|\iint_{[0,1]^{2}} C\left(\xi_{1}, \xi_{2}\right) d \xi_{1} d \xi_{2}\right| \leq \iint_{[0,1]^{2}} d \xi_{1} d \xi_{2}<+\infty
$$

and:

$$
\iint_{[0,1]^{2}}\left[C\left(\xi_{1}, \xi_{2}\right)\right]^{2} d \xi_{1} d \xi_{2} \leq \iint_{[0,1]^{2}} d \xi_{1} d \xi_{2}<+\infty
$$

Accordingly, the "process" that associates to any couple of random variables $\xi_{1}$ and $\xi_{2}$ uniformly distributed inside $[0,1]$ the random output $C\left(\xi_{1}, \xi_{2}\right)$ is of finite variance. In 
mathematical words, $C\left(\xi_{1}, \xi_{2}\right)$ is said to be second order. Then, following Cameron and Martin's theorem (see Ref. [14]), $C\left(\xi_{1}, \xi_{2}\right)$ can be expanded as an infinite series of orthogonal polynomials. This series converges towards the exact RV $C\left(\xi_{1}, \xi_{2}\right)$ in the $L^{2}$ sense. As the support of each variable $\xi_{1}$ and $\xi_{2}$ is [0,1], Ref. [9] (see Table 4.1 from this paper) suggests the shifted Legendre polynomials $P_{n}^{*}(\xi)$ (using the notations proposed in Ref. [18], in which further results about those polynomials can be found) to represent $C\left(\xi_{1}, \xi_{2}\right)$. Accordingly, we can write:

$$
C\left(\xi_{1}, \xi_{2}\right)=\sum_{n=0}^{+\infty} \sum_{m=0}^{+\infty} c_{n m} P_{n}^{*}\left(\xi_{1}\right) P_{m}^{*}\left(\xi_{2}\right)
$$

Coefficients $c_{n m}$ can be calculated by application of the orthogonality property of the polynomials (note that variables $\xi_{1}$ and $\xi_{2}$ are uncorrelated, by assumption) as:

$$
c_{n m}=\iint_{[0,1]^{2}} C\left(\xi_{1}, \xi_{2}\right) P_{n}^{*}\left(\xi_{1}\right) P_{m}^{*}\left(\xi_{2}\right) d \xi_{1} d \xi_{2}
$$

This infinite series expansion can be used to represent function $C\left(\xi_{1}, \xi_{2}\right)$ for any value of variables $\xi_{i} \in[0,1], i=1,2$.

However, in practice, it is not possible to estimate the infinite set of coefficients $c_{n m}$ and the double sum that appears in Eq. (11) has to be truncated at some finite integer order $N$. This means that we can "only" obtain in actual fact an approximation of $C$ as:

$$
C\left(\xi_{1}, \xi_{2}\right) \approx \sum_{n=0}^{N} \sum_{m=0}^{N} c_{n m} P_{n}^{*}\left(\xi_{1}\right) P_{m}^{*}\left(\xi_{2}\right)
$$

The accuracy of such a truncated series expansion will be studied later in this paper.

The main difficulty to apply Eqs. (11-13) is thus to calculate coefficients $c_{n m}$ as defined by Eq. (12). This problem is addressed in the next section.

\subsection{Evaluation of the coefficients in the series expansion}

From the previous developments, it would be at first sight natural to use the orthogonality property of shifted Legendre Polynomials - viz. to apply Eq. (12) directly - to estimate the coefficients in the series. However, we have chosen here to represent this series in terms of Cutteridge-Devyatov Polynomials (CDPs, see [19] and references therein for more details about those polynomials). As will be seen later, using this basis of polynomials allows several simplifications that are useful for practical applications. 
As shown in Ref. [19], any polynomial at an integer order $N$ defined over the interval $[0,1]$ can be written in terms of Cutteridge-Devyatov Polynomials (CDPs) $\Phi$. We can thus reformulate Eqs. $(11,12)$ using CDPs as:

$$
C\left(\xi_{1}, \xi_{2}\right) \approx C(0,0)+\sum_{n=0}^{N-1} \sum_{m=0}^{N-1} \chi_{n m} \Phi_{n}^{(N)}\left(\xi_{1}\right) \Phi_{m}^{(N)}\left(\xi_{2}\right)
$$

where [19]:

$$
\chi_{n m}=\iint_{[0,1]^{2}} \frac{\partial^{2} C\left(\xi_{1}, \xi_{2}\right)}{\partial \xi_{1} \partial \xi_{2}} \xi_{1}^{n} \xi_{2}^{m} d \xi_{1} d \xi_{2}
$$

It can be readily shown (see Appendix for details) that those coefficients can be rewritten as:

$$
\chi_{n m}=\iint_{[0,1]^{2}}\left\{\frac{\partial \alpha_{1}^{\Delta \eta}\left[l_{1}\left(\xi_{1}\right)\right]}{\partial L_{1}} \frac{\partial \alpha_{2}^{\Delta \eta}\left[l_{2}\left(\xi_{2}\right)\right]}{\partial L_{2}}\right\}^{-1} \frac{\partial^{2} \Gamma_{12}^{\Delta \eta}\left[l_{1}\left(\xi_{1}\right), l_{2}\left(\xi_{2}\right)\right]}{\partial L_{1} \partial L_{2}} \xi_{1}^{n} \xi_{2}^{m} d \xi_{1} d \xi_{2}
$$

Coefficients $\chi_{n m}$ can thus be calculated directly from LBL data as the application of Eq. (16) only requires evaluating narrow band averaged absorptivities of uniform and non-uniform paths. This can be readily done as soon as LBL data are available.

Furthermore, the first term at the RHS in the second equation is null since, from Eqs. $(6,8)$, $C(0,0)=\Gamma_{12}^{\Delta \eta}\left(L_{1}=0, L_{2}=0\right)=0$.

Representation (14) will be referred to as the Cutteridge-Devyatov Polynomial Chaos (CDPC) expansion of $C$ at order $N$ in the following.

A simple and accurate method to handle the calculation of coefficients $\chi_{n m}$ from LBL data by a direct application of Eq. (16) is provided in Section 3.

\subsection{Mathematical analysis of the role played by function $C$ in the treatment of spectral correlations}

In order to explain why it is possible to treat spectral correlations using the series expansion (11), let us focus on function $C:(u, v) \in[0,1]^{2} \rightarrow[0,1]$ in which $u$ and $v$ are real numbers. Following Eq. (11), this function is defined as:

$$
C(u, v)=\sum_{n=0}^{+\infty} \sum_{m=0}^{+\infty} c_{n m} P_{n}^{*}(u) P_{m}^{*}(v)=\Gamma_{12}^{\Delta \eta}\left[l_{1}(u), l_{2}(v)\right]
$$

in which the coefficients $c_{n m}$ remain the same as in Eq. (12).

We can apply this function to the couple of deterministic inputs $u=\alpha_{1}^{\Delta \eta}\left(L_{1}\right)$ and $v=\alpha_{2}^{\Delta \eta}\left(L_{2}\right)$. In this case, from the definition of functions $l_{i}, i=1,2$ (see Eq. (7)), it follows 
that $l_{1}(u)=l_{1}\left[\alpha_{1}^{\Delta \eta}\left(L_{1}\right)\right]=L_{1}$ and $l_{2}(v)=l_{2}\left[\alpha_{2}^{\Delta \eta}\left(L_{2}\right)\right]=L_{2}$. We can now replace those quantities inside Eq. (17) and write:

$$
\Gamma_{12}^{\Delta \eta}\left(L_{1}, L_{2}\right)=\sum_{n=0}^{+\infty} \sum_{m=0}^{+\infty} c_{n m} P_{n}^{*}\left[\alpha_{1}^{\Delta \eta}\left(L_{1}\right)\right] P_{m}^{*}\left[\alpha_{2}^{\Delta \eta}\left(L_{2}\right)\right]
$$

Eq. (18), together with Eq. (5), thus provides an exact way to handle the calculation of the absorptivity over the non-uniform paths $L_{1}+L_{2}$ (what we usually want to estimate in many applications) from the values of absorptivities over each of the uniform sub-paths $L_{i}, i=1,2$ (quantities that can be evaluated using any approximate model for uniform media):

$$
\begin{aligned}
\alpha_{12}^{\Delta \eta}\left(L_{1}, L_{2}\right)= & \alpha_{1}^{\Delta \eta}\left(L_{1}\right)+\alpha_{2}^{\Delta \eta}\left(L_{2}\right) \\
& -\sum_{n=0}^{+\infty} \sum_{m=0}^{+\infty} c_{n m} P_{n}^{*}\left[\alpha_{1}^{\Delta \eta}\left(L_{1}\right)\right] P_{m}^{*}\left[\alpha_{2}^{\Delta \eta}\left(L_{2}\right)\right]
\end{aligned}
$$

The simplest way to understand why spectral correlation can be handled by the previous series expansion relies on copula's theory. It is out of the scope of the present work to provide a comprehensive depiction of this topic. Interested readers should refer, for instance, to Refs. [20,21] for additional details.

A copula is a mathematical tool that enables to analyse and/or model the dependency between random variables. It is not surprising to see this concept appear in the frame of gas radiation as the name itself "spectral correlation" suggests it.

A bivariate copula $C:(u, v) \in[0,1]^{2} \rightarrow[0,1]$ is mathematically defined [21] as a function that links a bivariate distribution to its one-dimensional marginal distribution functions. It has the following properties ([21], p. 10):

P3/

$$
\text { for every } u, v \in[0,1], C(u, 0)=C(0, v)=0
$$

P4I

$$
\text { for every } u, v \in[0,1], \mathrm{C}(u, 1)=u, \mathrm{C}(1, v)=v
$$

$P 5 /$ for $0 \leq u_{1} \leq u_{2} \leq 1 \quad$ and $0 \leq v_{1} \leq v_{2} \leq 1, \quad C\left(u_{2}, v_{2}\right)-C\left(u_{2}, v_{1}\right)-C\left(u_{1}, v_{2}\right)+C\left(u_{1}, v_{1}\right) \geq 0$

It was shown by Sklar [22] that for any bivariate distribution $H(x, y)$ with marginals $F(x)$ and $G(y), x, y \in[-\infty,+\infty]$ there exists a copula $\bar{C}$ such that:

$$
H(x, y)=\bar{C}[F(x), G(y)]
$$

Furthermore, if the marginals are continuous, the copula is unique. Reciprocally, if $F(x)$ and $G(y)$ are two univariate distribution functions and $\bar{C}$ is a copula then $H(x, y)$ is a bivariate distribution. 
It can be readily checked, by a direct application of the definition of function $\Gamma_{12}^{\Delta \eta}\left(L_{1}, L_{2}\right)$ (see Eq. (6)), that function $C$ as defined by Eq. (17) is a copula. Indeed (using the same notations as in the previous section):

P3/

$$
\begin{aligned}
& C(u, v=0)=\Gamma_{12}^{\Delta \eta}\left(L_{1}, L_{2}=0\right)=0 \\
& C(u=0, v)=\Gamma_{12}^{\Delta \eta}\left(L_{1}=0, L_{2}\right)=0
\end{aligned}
$$

$P 4 /$

$$
\begin{aligned}
& C(u, v=1)=\Gamma_{12}^{\Delta \eta}\left(L_{1}, L_{2}=+\infty\right)=\alpha_{1}^{\Delta \eta}\left(L_{1}\right)=u \\
& C(u=1, v)=\Gamma_{12}^{\Delta \eta}\left(L_{1}=+\infty, L_{2}\right)=\alpha_{2}^{\Delta \eta}\left(L_{2}\right)=v
\end{aligned}
$$

P5/

see Appendix

Accordingly, the series expansion Eq. (17) is the unique representation of $C$ (it is unique because the marginals are in our case the $\ell$-distributions, viz. the narrow band averaged absorptivities over the uniform sub-paths, which are obviously continuous with respect to the gas path lengths). This means that the polynomial approximation set by Eq. (14), which was obtained through an input-output stochastic modeling using a PC expansion, is also a nonparametric estimate of this copula. This also explains why the non-uniform approximation provided by Eq. (18) - and approximated by Eq. (14) - enables to handle spectral correlations: the aim of copulas is precisely to model such a dependency.

\section{APPLICATION}

\subsection{LBL data and model parameters}

All the test cases considered in the present work are for $\mathrm{H}_{2} \mathrm{O}-\mathrm{N}_{2}$ mixtures only. Indeed, as the method provides a way to estimate absorptivities of non-uniform paths averaged over narrow bands, the technique can be extended to mixtures by assuming, as usual [1-2,8], spectra of distinct absorbing molecular species to be uncorrelated. Accordingly, mixtures of absorbing species will not be considered here.

The LBL data used as references consist of high resolution $\left(10^{-2} \mathrm{~cm}^{-1}\right)$ spectra based on the HITEMP2010 spectroscopic database [23]. Additional details about these reference spectra (partition functions, evaluation of line profiles, etc) can be found in Ref. [10]. They are not reported again here.

The steps proposed to evaluate the coefficients $\chi_{n m}$ required for the use of Eq. (14) are the following ones:

$1 /$ evaluate the weights $\left(\omega_{i}, i=1, . ., N\right)$ and abscissas $\left(\xi_{i}, i=1, . ., N\right)$ associated with the Gauss Legendre quadrature translated to the interval [0,1] - order $N=20$ was chosen in this work. The subroutine to evaluate those coefficients was taken from Ref. [24].

2/ for each thermophysical state $\phi_{i}, i=1,2$, calculate the narrow band absorptivities by applying their definition Eq. (2) to the LBL dataset. This process is done for $10^{4}$ values of parameter $L_{i}$ (see Eq. (2)) logarithmically scaled between $10^{-5} \mathrm{~cm}$ and $10^{8} \mathrm{~cm}$. 
3/ for each set of abscissas $\xi_{j}, j=1, . ., N$, evaluate the corresponding lengths $l_{i}\left(\xi_{j}\right), i=1,2, j=1, . ., N$ by solving the implicit equation set by Eq. (7). These solutions are estimated directly by interpolating (logarithmically with respect to $L$ ) between the two closest values of narrow band absorptivities found at the second step of the process.

4/ then, evaluate the derivatives $\partial \alpha_{1}^{\Delta \eta}\left[L_{1}=l_{1}\left(\xi_{i}\right)\right] / \partial L_{1}, \partial \alpha_{2}^{\Delta \eta}\left[L_{2}=l_{2}\left(\xi_{j}\right)\right] / \partial L_{2}$ (see Eq. (3)) and $\frac{\partial^{2} \Gamma_{12}^{\Delta \eta}\left[L_{1}=l_{1}\left(\xi_{i}\right), L_{2}=l_{2}\left(\xi_{j}\right)\right]}{\partial L_{1} \partial L_{2}}$ (more details about the calculation of this derivative are given in Appendix) for $i=1, . ., N$ and $j=1, . ., N$.

5/ estimate the coefficients $\chi_{n m}$ by application of the following formula (which is the discrete form of Eq. (16)):

$$
\chi_{n m}=\sum_{i=1}^{N} \sum_{j=1}^{N} \omega_{i} \omega_{j} \xi_{i}^{n} \xi_{j}^{m}\left\{\frac{\partial \alpha_{1}^{\Delta \eta}\left[l_{1}\left(\xi_{i}\right)\right]}{\partial L_{1}} \cdot \frac{\partial \alpha_{2}^{\Delta \eta}\left[l_{2}\left(\xi_{j}\right)\right]}{\partial L_{2}}\right\}^{-1} \frac{\partial^{2} \Gamma_{12}^{\Delta \eta}\left[l_{1}\left(\xi_{i}\right), l_{2}\left(\xi_{j}\right)\right]}{\partial L_{1} \partial L_{2}}
$$

The full process (starting from the second step up to the application of those coefficients for the calculation of the band averaged absorptivity of a non-uniform path) is summarized in Figure 1. It can be noticed that coefficients $\chi_{n m}$ do not depend on the gas path lengths. They can thus be evaluated once and then stored in tables for later use.

As soon as coefficients $\chi_{n m}$ are known (up to a prescribed order $N, 0 \leq n, m \leq N$ ), they can be used inside Eq. (14). Univariate CDPs are then polynomials at order $N$. Results of comparisons are given in the next section.

\subsection{Application}

To illustrate the validity of the present method, several line-of-sight ( 0 dimensional) examples will be considered. In all cases (except case C5 described below), a slab of a gas at high temperature radiates through a cold gas at a low temperature. Both columns are at atmospheric pressure. Even though the situation is simple from a geometrical perspective, those cases are known to be difficult to handle using usual models. Indeed, they were all introduced by their respective authors to assess some sophisticated techniques such as: $1 /$ the multigroup approach by Modest and co-workers [25] (see test case C1 below), 2/ the SNB-FG (Statistical Narrow Band model with the Fictitious Gas approximation) model by Soufiani et al. [26] (case C2); or to illustrate some limits of "simple" non-uniform approximations [4] (Case C3). Some other examples are also studied that show that the present approach is general. For this purpose, we have considered two other cases: $\mathrm{C} 4$ for which the temperature gradient is small $(200 \mathrm{~K})$ and $\mathrm{C} 5$ for which the two columns are at the same temperature but the species concentrations are different in the two layers. In these last two kinds of situations, the correlated assumption is known to provide accurate results. 


\subsubsection{Description of the test cases}

Case C1 was proposed in Ref. [25]. Both layers are at the same total and partial pressures of $\mathrm{H}_{2} \mathrm{O}$ (molar fraction in water vapor is 0.2 ). The two slabs have the same length $L_{1}=L_{2}=50$ $\mathrm{cm}$. The temperatures of the cold and hot layers are $300 \mathrm{~K}$ and $2000 \mathrm{~K}$ respectively. Results, provided in terms of emissivity as defined by Modest in Ref. [27], are depicted in Figure 2 for the $6.3 \mu \mathrm{m}$ band of water vapor.

Case C2 was described in Ref. [26]. It is representative of a plume signature calculation. The two slabs have distinct lengths (100 $\mathrm{cm}$ for the hot column, $500 \mathrm{~m}$ for the cold one), temperature $(300 \mathrm{~K}$ and $2100 \mathrm{~K})$ and $\mathrm{H}_{2} \mathrm{O}$ molar fractions $(0.01$ in the cold layer, 0.08 in the hot one). Results, provided in terms of radiative intensities, are shown in Figure 3 for the same $6.3 \mu \mathrm{m}$ band of water vapor as for case $\mathrm{C} 1$.

The next case, C3, was proposed in Ref. [4] to compare various methods (Curtis-Godson, Lindquist-Simmons) to extend SNB models from uniform to non-uniform media. It was found in this reference that none of the existing technique was able to treat this situation accurately. However, this allowed the authors to conclude that among those possibilities, the CG approximation was the best, though inaccurate, one.

Here, the cold and hot layers have a length of $10 \mathrm{~cm}$ and $100 \mathrm{~cm}$ respectively. The hot gas is at $1500 \mathrm{~K}$ and the cold one at $500 \mathrm{~K}$. The $\mathrm{H}_{2} \mathrm{O}$ molar fraction is 0.05 over the cold path and 0.5 in the hot region. Results are depicted, in terms of radiative intensities, in Figure 4. The spectral interval now extends from $1200 \mathrm{~cm}^{-1}$ up to $1900 \mathrm{~cm}^{-1}$ to comply with the figure provided in Ref. [4]. Units for LBL intensities are also the same as used in this reference.

Case $\mathrm{C} 4$ is the same as $\mathrm{C} 1$ but the hot layer is at $500 \mathrm{~K}$. Results are shown in Figure 5.

Case C5 is the same as C1 but both layers are at $2000 \mathrm{~K}$. The molar fraction in the first layer is 0.1 and 0.2 in the second one. Results are plotted in Figure 6.

Each Figure (2-6) consists of the same set of curves. In fact, on each of them, we have reported results (in terms of emissivity or radiative intensity) for several approximate models. At the top, LBL calculations, used as references, are plotted together with the Uncorrelated approximation and C- $k$ models. The Uncorrelated approximation corresponds to the following estimate:

$$
\Gamma_{12, \text { uncorrelated }}^{\Delta \eta}\left(L_{1}, L_{2}\right)=\frac{1}{\Delta \eta} \int_{\Delta \eta}\left[1-\exp \left(-\kappa_{\eta, 1} L_{1}\right)\right] d \eta \times \frac{1}{\Delta \eta} \int_{\Delta \eta}\left[1-\exp \left(-\kappa_{\eta, 2} L_{2}\right)\right] d \eta
$$

As shown in Appendix, it corresponds to the CDPC approximation at order 1.

The C- $k$ model was built with the same LBL data chosen as references. The quadrature described in Section 3.1. (Gauss Legendre at order 20) was used to discretize the $g$-space. This $k$-distribution model is almost exact (when compared to LBL calculations) in uniform situations.

At the bottom of the Figures, we have depicted the relative errors for several models when compared to LBL results for non-uniform calculations. The first one is the $k$-distribution model based on the assumption of correlated spectra $(\mathrm{C}-k)-$ it is the same C-k model as 
shown at the top of the figures; the next three curves correspond to the CDPC model proposed in this work with three polynomial orders (for each univariate set): 2 (CDPC at order 2), 3 (CDPC at order 3) and 9 (CDPC at order 9). As explained in Appendix (see Eq. (A.14)), the CDPC model at order 2 requires to evaluate only 1 coefficient $\left(\chi_{11}\right)$. The number of coefficients $\chi_{n m}$ required - per narrow band - for the calculations is 4 at order 3 and 64 at order 9. As LBL and $k$-distributions were found to yield exactly the same results in uniform situations (relative errors of the order of $0.1 \%$ ), and as our approach only requires the values of the absorptivities over uniform paths, LBL absorptivities were used in the CDPC calculations.

\subsubsection{Analysis of the results and discussion}

From the analysis of those Figures, the following comments can be drawn:

- as expected, the uncorrelated approximation is the one that usually performs the worst. The correlated approximation is accurate in cases $\mathrm{C} 4$ and $\mathrm{C} 5$ but fails to provide reliable estimates in Cases C1-3.

- the accuracy of the bivariate polynomial approximation increases with the order of those polynomials. At an order $N=9$ in each variable, which is the maximum value considered in the present work, the method achieves almost LBL accuracy. Furthermore, it can be noticed that although low order approximations at $N=2$ and $N=3$ do not allow very accurate estimates of the radiative quantities at a narrow band scale, their accuracy - when the data are averaged over wide bands - are in most cases higher (C1-3) or similar (C4-5) to those obtained by the usual C- $k$ approach (see Table 1 - it corresponds to relative errors, in $\%$, calculated as $100 \mathrm{x}(1-$ Model / LBL). The same spectra and wavenumber ranges as plotted in Figures 2-6 were used. Data were averaged over these spectral intervals - emissivities were converted to intensities before to perform the calculations of those relative errors). Indeed, C$k$ model provides relative errors that can reach $26.7 \%$ when compared to LBL calculations when the CDPC approach at order 2 does not exceed $6.2 \%$. Moreover, relative errors for the CDPC model at order 3 are in any case lower than $1.2 \%$. When order 9 is considered, the approach achieves almost LBL accuracy (relative error is lower than $0.1 \%$ for all the test cases considered here).

In order to investigate this result further, we have considered three additional test cases derived from Cases $\mathrm{C} 1$ and $\mathrm{C} 2$. However, instead of keeping the length of the cold path constant, this quantity is allowed to increase from $0 \mathrm{~cm}$ up to $150 \mathrm{~cm}$ (for Case C1) and from $500 \mathrm{~cm}$ up to $10^{6} \mathrm{~cm}$ (for Case C2). Furthermore, results of the various models (radiative intensities) are calculated over each narrow band and then averaged over the full spectrum. Only the CDPC model at order 2 (which consists of a single coefficient, as noticed in Appendix) is considered. Results are plotted in Figure 7 (same as C1), Figure 8 (the temperature of the hot gas is $1000 \mathrm{~K}$ ), and Figure 9 (case $\mathrm{C} 2$ - this figure can be compared directly with Figure 6 from Ref. [26]).

It can be noticed that results combining the CDPC model at order 2 with absorptivities of uniform paths calculated by a $k$-distribution approach are also given. As expected, there is no significant difference between this model and the one based on absorptivities calculated LBL (as used in Figures 2-6). 
From Figures 7-9, it is clear that using the CDPC model at order 2 provides more accurate results than the $\mathrm{C}-k$ model for calculations over the full spectrum. This confirms the results given in Table 1.

Furthermore, in these cases - that can be considered as extreme in terms of spectral correlation -, our approach enables to achieve an accuracy that is comparable to both the so-called Multigroup and CKFG approaches. This means that using any accurate model for narrow band absorptivities over uniform paths and adding just one coefficient (for a couple of thermophysical states) in our method one can obtain results as precise as more sophisticated techniques for heat transfer applications. The present method thus outperforms many existing models without requiring any simplifying assumption (such as scaling or correlation of gas spectra). The main source of error arises from the order of the series expansion. Order 2 provides accurate results for the evaluation of radiative quantities averaged over the full spectrum.

However, all the calculations considered in this work were for only two uniform sub-paths. In many applications, a higher number of such sub-paths is required. In these situations, all the theoretical developments provided in this work remain correct. The main difficulty to extend the present approach to more general radiative transfer calculations is practical. However, as explained below, many possible techniques can be used to solve this problem.

\subsection{Extension of the approach to dimensions higher than 2}

Many papers have been devoted to the generalization of gPC expansions to high dimensions. Indeed, the main difficulty for the extension of the present method to more than two RVs is that the number of sums (see for instance Eq. (14)) increases exponentially with the number of independent RVs (ie in our case of uniform sub-paths) in the parametrization. This implies both a significant growth in terms of number of coefficients to estimate but also in terms of the computational cost for the numerical evaluation of the multivariate polynomials. This issue is referred to as the curse of dimensionality [15]. However, several techniques have been proposed in the literature to handle this problem.

In Ref. [28], for instance, gPC is extended up to a dimension (that would correspond, in our case, to a number of distinct thermophysical states) equal to 100 using a sparse PC expansion. The method proposed in this reference is likely to enable the extension of this work to more general radiative heat transfer problems.

Another possible approach consists in approximating the high dimensional function $C$ using the same technique as used in the Random Sampling - High Dimensional Model Representation method (RS-HDMR) [29]. This approach, mostly based on an expansion of the function into correlated components of increasing orders, allows approximating high dimensional functions (dozens of random input variables can be handled without any neither mathematical nor technical difficulties). The principle of this technique is almost the same as gPC, but written in a slightly different way [16,29].

The two previous techniques are funded on the same principle that consists in modeling stochastically some input-output relationship. This was also the approach chosen in this work.

Another possibility would consist in handling the problem directly in terms of distribution functions viz. through copula models - a compendium of the existing ones can be found in Refs. [20,21]. Then, the difficulty consists of finding which copula model to use. However, as 
noticed in Appendix, copula models based on the so-called FGM (Farlie-GumbelMorgenstern, sometimes referred to as the Eyraud-Gumbel-Morgenstern, EGM, or EyraudFarlie-Gumbel-Morgenstern, EFGM) family [20] appears to be a relevant choice to initiate such a study. A generalization of the FGM family to multivariate distributions can be found in Ref. [30].

Evaluating all those methods in the frame of gas radiation in non-uniform media is scheduled as future work.

However, it should be noticed that the present technique does not have - a priori - for objective to replace general purpose approaches, such as the C- $k$ model, for heat transfer applications. But it is a relevant candidate for applications that require a high accuracy at narrow band scales such as encountered in quantitative spectroscopic analysis (in combustion or atmospheric sciences).

\section{CONCLUSION}

A method was proposed for the approximate modeling of the radiative properties of nonuniform gaseous media. The mathematical derivation was described. It relies on the fact that narrow band averaged absorptivities are $\ell$-distribution functions. The polynomial chaos expansion of the absorptivity of a non-uniform path was performed. The corresponding series expansion was shown to correspond to a copula. The copula enables to account for the spectral correlation between spectra associated with gases in distinct thermophysical states. As the infinite series expansion can only be approximated, low order approximations (at orders 2, 3 and 9) were considered. A simple method to estimate the coefficients in the series was provided. In contrast with usual models, the approach does not require any simplifying assumption (of scaling or correlation of gas spectra). Its main source of inaccuracy arises from the order chosen to approximate the series expansion. The non-uniform model was shown to be very accurate: at order 9, it achieves almost LBL accuracy. At order 2, the method was shown to outperform the usual $\mathrm{C}-k$ model for full spectrum radiative heat transfer calculations. Extension of the technique to higher dimensions was discussed and is planned as future work.

\section{REFERENCES}

[1] GOODY R. M., YUNG Y. L. Atmospheric Radiation: Theoretical Basis, Oxford University Press, 1989.

[2] TAINE J, SOUFIANI A. Gas IR radiative properties: From spectroscopic data to approximate models, Adv. Heat Transfer 1999;33:295-414.

[3] MALKMUS W. Random Lorentz band model with exponential-tailed S-1 line-intensity distribution function, J. Opt. Soc. Am., Vol. 57, pp 323-329, 1967.

[4] SOUFIANI A., HARTMANN J. M., TAINE J. Validity of band-model calculations for $\mathrm{CO}_{2}$ and $\mathrm{H}_{2} \mathrm{O}$ applied to radiative properties and conductive-radiative transfer, JQSRT, Vol. 33, pp 243-257, 1985.

[5] AMBARTZUMIAN V. The effect of the absorption lines on the radiative equilibrium of the outer layers of the stars, Publ. Obs. Astron. Univ. Leningrad, Vol. 6, pp 7-18, 1934. 
[6] YOUNG S.J. Band model formulation for inhomogeneous optical paths, JQSRT 1975;15:483-501.

[7] LACIS A.A., OINAS V. A description of the correlated $\mathrm{k}$ distribution method for modeling non-gray gaseous absorption, thermal emission, and multiple scattering in vertically inhomogeneous atmospheres, J. Geophys. Rev. D., Vol. 96, pp 9027-9063, 1991.

[8] MODEST M.F. Radiative heat transfer, $2^{\text {nd }}$ ed. New York: Academic Press; 2003 (ISBN 0-12-503163-7).

[9] XIU D, KARNIADAKIS G.M. The Wiener-Askey polynomial chaos for stochastic differential equations, SIAM J. Sci. Comput. 2002;24:619-644.

[10] ANDRE F., HOU L., SOLOVJOV V.P., GALTIER M. An exact $k$-distribution formulation for Radiative Heat Transfer in non-uniform gaseous media. Submitted to JQSRT, 2015.

[11] GAUTSCHI W. Orthogonal polynomials - computation and approximation, Oxford Science Publishing, 2004.

[12] CHIHARA T.S. An introduction to orthogonal polynomials, Dover; 1978 (ISBN-10: 0486-47929-3).

[13] WIENER N. The Homogeneous Chaos, American Journal of Mathematics 1938;60:897936.

[14] CAMERON R.H., MARTIN W.T. The orthogonal development of non-linear functional in series of Fourier-Hermite functional, Annals of Mathematics, Second series, 1947;48:385392.

[15] LE MAITRE O.P., KNIO O.M. Spectral methods for uncertainty quantification - with applications to computational fluid dynamics, Springer, 2010

[16] MUNOZ ZUNIGA M., KUCHERENKO S., SHAH N. Metamodelling with independent and dependent inputs, Comp. Phys. J. 2013;184:1570-1580.

[17] SCHORACK G.R. Probability for statisticians, Springer texts in statistics, Springer, 2000 .

[18] ABRAMOWITZ M., STEGUN I.A. Handbook of Mathematical Functions with formulas, Graphs, and Mathematical Tables, New York: Dover 1965.

[19] ANDRE F., GALTIER M. On the use of polynomial chaos expansions and generalized moments within the frame of gas radiation in non-uniform gaseous media, JQSRT 2015;164:184-192.

[20] NELSEN R.B. An introduction to Copulas - Second Edition, Springer series in statistics, Springer, 2006.

[21] BALAKRISHNAN N., LAI C-D. Continuous bivariate distributions - Second edition, Springer, 2009. 
[22] SKLAR A. Fonctions de répartitions et leurs marges, Publications of the Institute of Statistics, Université de Paris, 1959;8:229-231.

[23] ROTHMAN L.S., GORDON L.E., BARBER R.J., DOTHE H, GAMACHE R.R., GOLDMAN A, PEREVALOV V.I., TASHKUN S.A., TENNYSON J. HITEMP, the hightemperature molecular spectroscopic database, JQSRT 2010;111:2139-2150.

[24] Numerical recipes in Fortran 77: the art of scientific computing. Cambridge: Cambridge University Press; 1992.

[25] ZHANG H, MODEST M.F. Multi-group full-spectrum $k$-distribution database for water vapor mixtures in radiative transfer calculations, IJHMT 2003;46:3593-3603.

[26] SOUFIANI A, ANDRE F, TAINE J. A fictitious-gas based statistical narrow-band model for IR long-range sensing of $\mathrm{H}_{2} \mathrm{O}$ at high temperature, JQSRT $2002 ; 73$ :339-347.

[27] MODEST M.F. Narrow-band and full-spectrum $k$-distributions for radiative heat transfer - correlated- $k$ vs. scaling approximation, JQSRT 2003;76:69-83.

[28] BLATMAN G., SUDRET B. Adaptative sparse polynomial chaos expansions based on Least Angle Regression, J. Comp. Phys. 2011;230:2345-2367.

[29] LI G., WANG S.-W., RABITZ H. Practical approaches to construct RS-HDMR component functions, J. Phys. Chem. 2002;106:8721-8733.

[30] CAMBANIS S. Some properties and generalizations of multivariate Eyraud-GumbelMorgenstern distributions, J. Multivariate Analysis 1977;7:551-559.

[31] ANDRE F., SOLOVJOV V.P., HOU L., VAILLON R., LEMONNIER D. The generalized $k$-moment method for the modeling of cumulative $k$-distributions of $\mathrm{H}_{2} \mathrm{O}$ at high temperature, JQSRT $2014 ; 143$ :92-99. 


\section{FIGURE CAPTIONS}

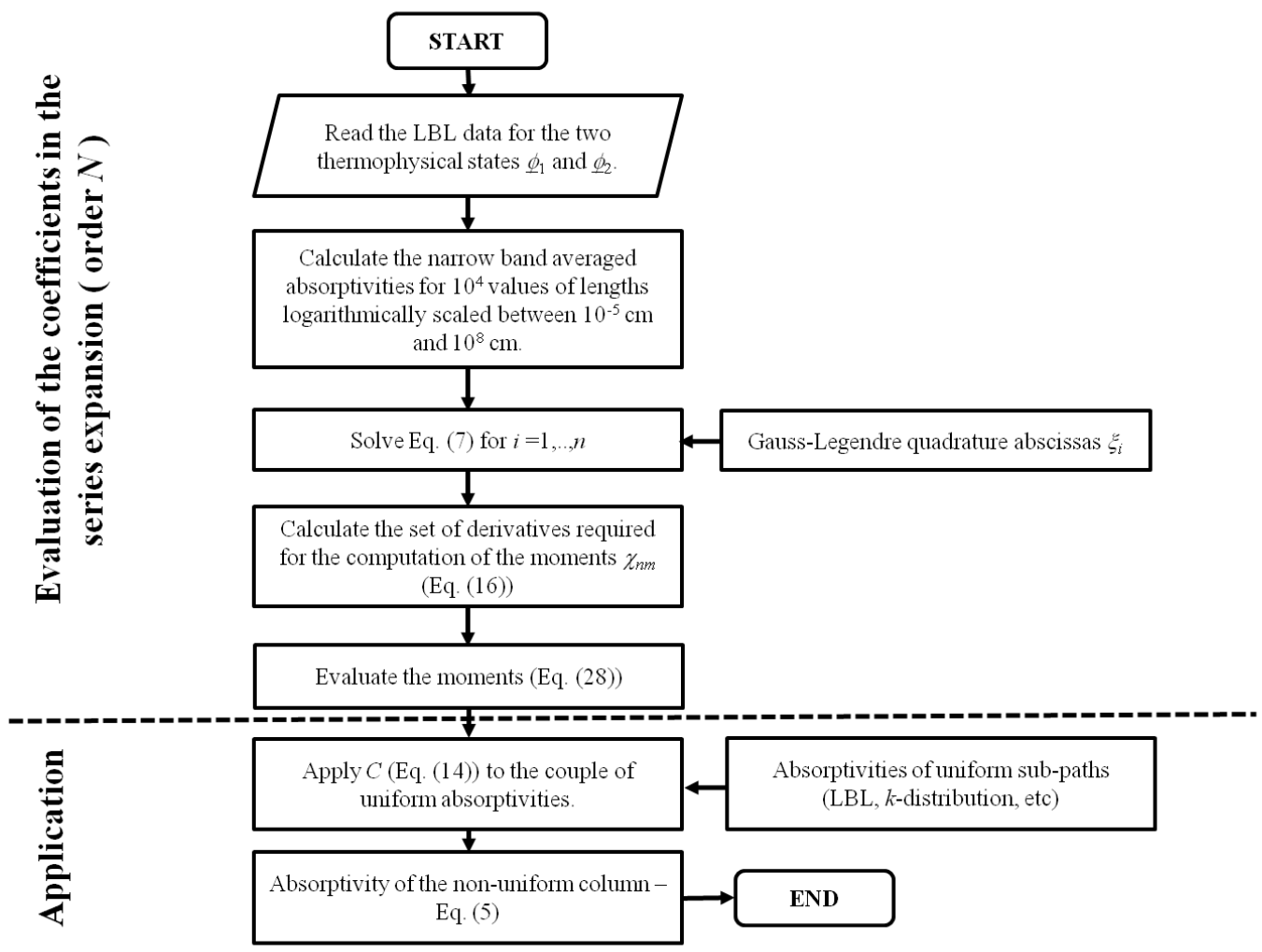

Figure 1. Flowchart for building coefficients in the series expansion and apply them to estimate the absorptivity of a non-uniform path. 


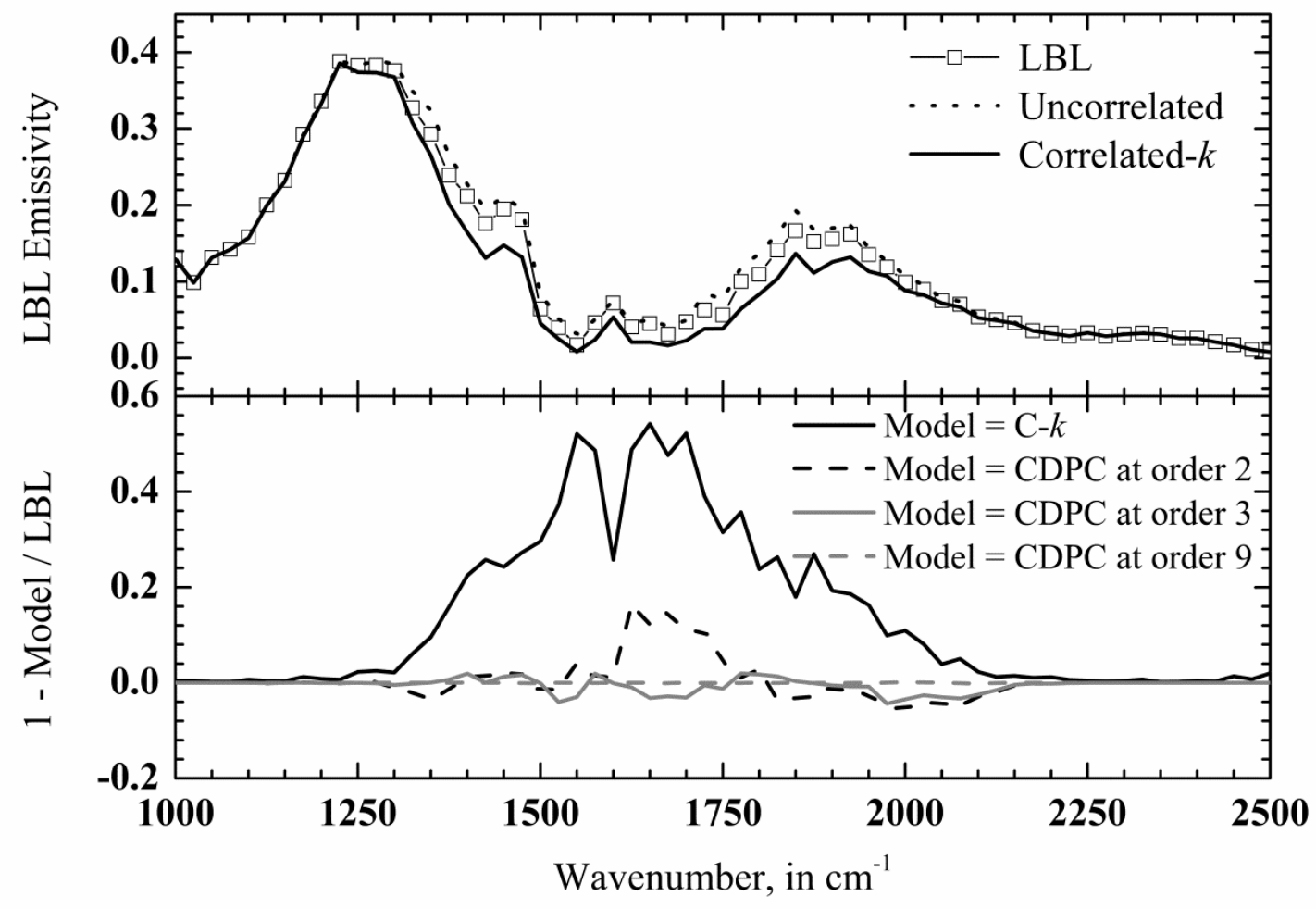

Figure 2. Narrow band emissivity for the $6.3 \mu \mathrm{m}$ band $-\left[1000 \mathrm{~cm}^{-1} ; 2500 \mathrm{~cm}^{-1}\right]-$ of $\mathrm{H}_{2} \mathrm{O}$ calculated by the LBL, uncorrelated, C- $k$ and CDPC (at orders 2, 3 and 9) models. $20 \% \mathrm{H}_{2} \mathrm{O}$ and $80 \% \mathrm{~N}_{2}$ at 1 atmosphere. The temperature (lengths) of the cold and hot paths are $300 \mathrm{~K}$ $(50 \mathrm{~cm})$ and $2000 \mathrm{~K}(50 \mathrm{~cm})$ respectively. 


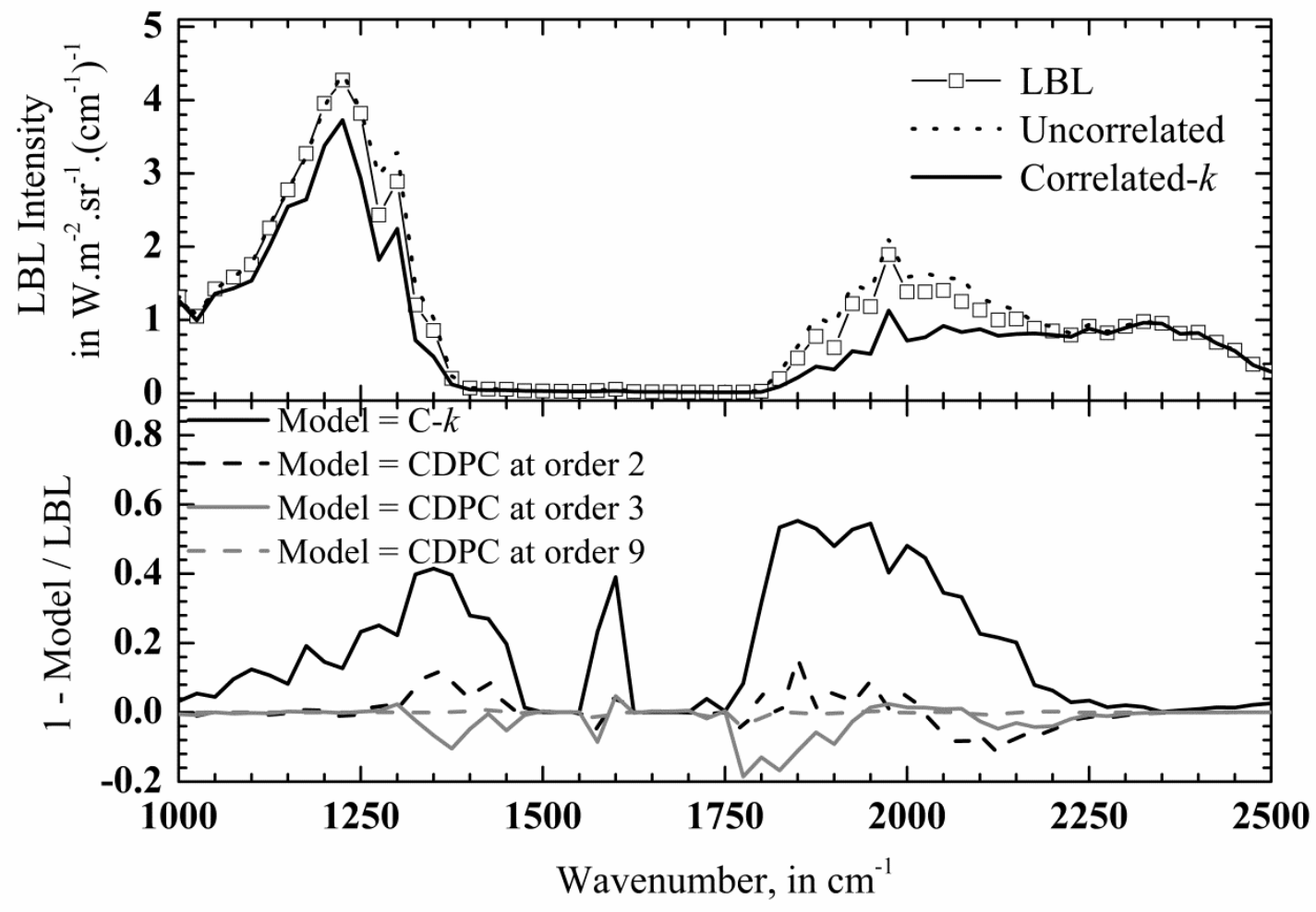

Figure 3. Narrow band radiative intensity for the $6.3 \mu \mathrm{m}$ band $-\left[1000 \mathrm{~cm}^{-1} ; 2500 \mathrm{~cm}^{-1}\right]$ - of $\mathrm{H}_{2} \mathrm{O}$ calculated by the LBL, uncorrelated, C- $k$ and CDPC (at orders 2,3 and 9 ) models. $1 \%$ $\mathrm{H}_{2} \mathrm{O}$ and $99 \% \mathrm{~N}_{2}$ at 1 atmosphere in the cold column. $8 \% \mathrm{H}_{2} \mathrm{O}$ and $92 \% \mathrm{~N}_{2}$ at 1 atmosphere in the hot column. The temperature of the cold and hot paths are $300 \mathrm{~K}(500 \mathrm{~m})$ and $2100 \mathrm{~K}$ $(100 \mathrm{~cm})$ respectively. 


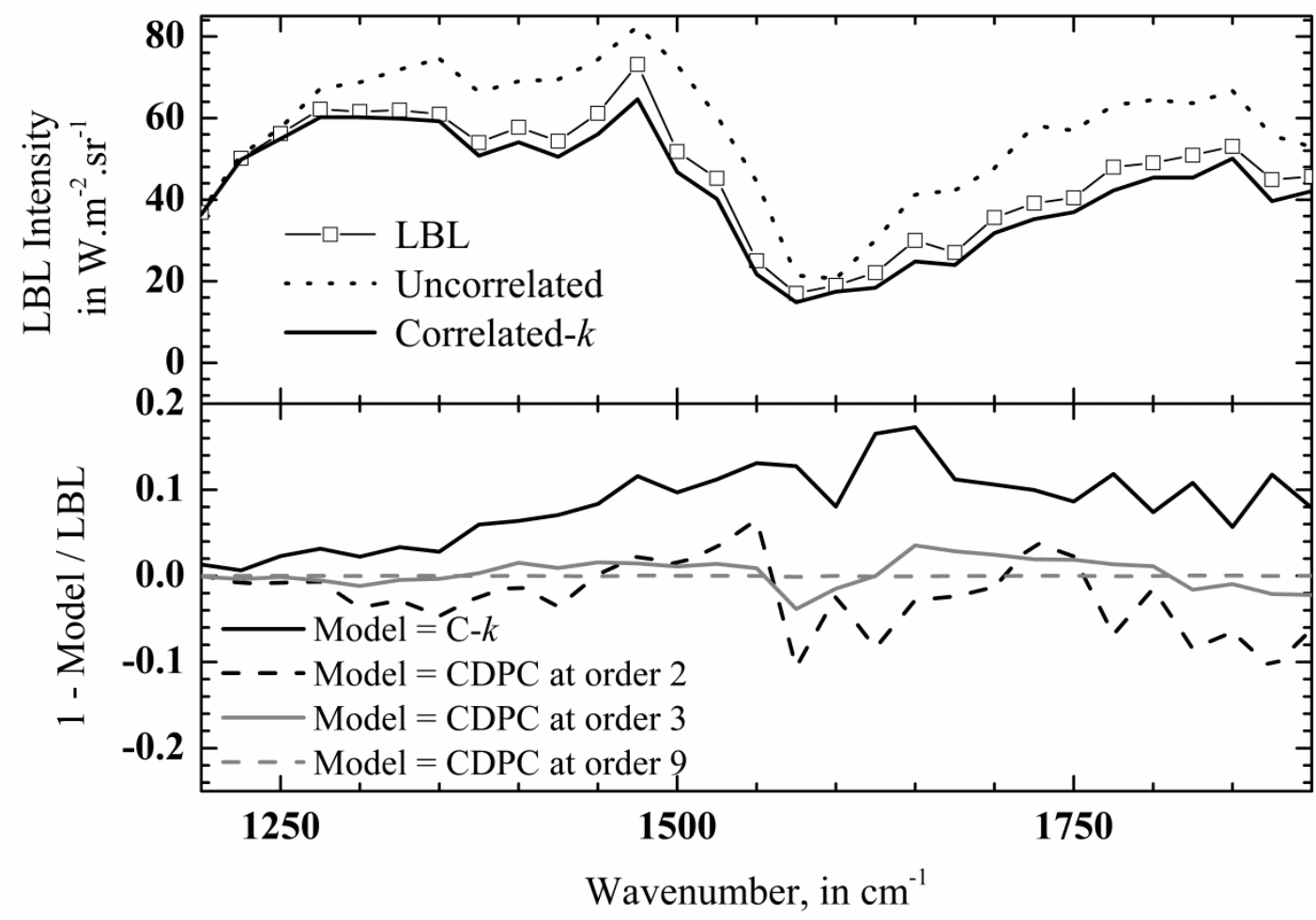

Figure 4. Narrow band radiative intensity for the $6.3 \mu \mathrm{m}$ band of $\mathrm{H}_{2} \mathrm{O}$ calculated by the LBL, uncorrelated, C- $k$ and CDPC (at orders 2, 3 and 9) models. $5 \% \mathrm{H}_{2} \mathrm{O}$ and $95 \% \mathrm{~N}_{2}$ in the cold layer; $50 \% \mathrm{H}_{2} \mathrm{O}$ and $50 \% \mathrm{~N}_{2}$ in the hot layer; both layers are at 1 atmosphere. The temperature of the cold and hot paths are $500 \mathrm{~K}(100 \mathrm{~cm})$ and $1500 \mathrm{~K}(10 \mathrm{~cm})$ respectively. 


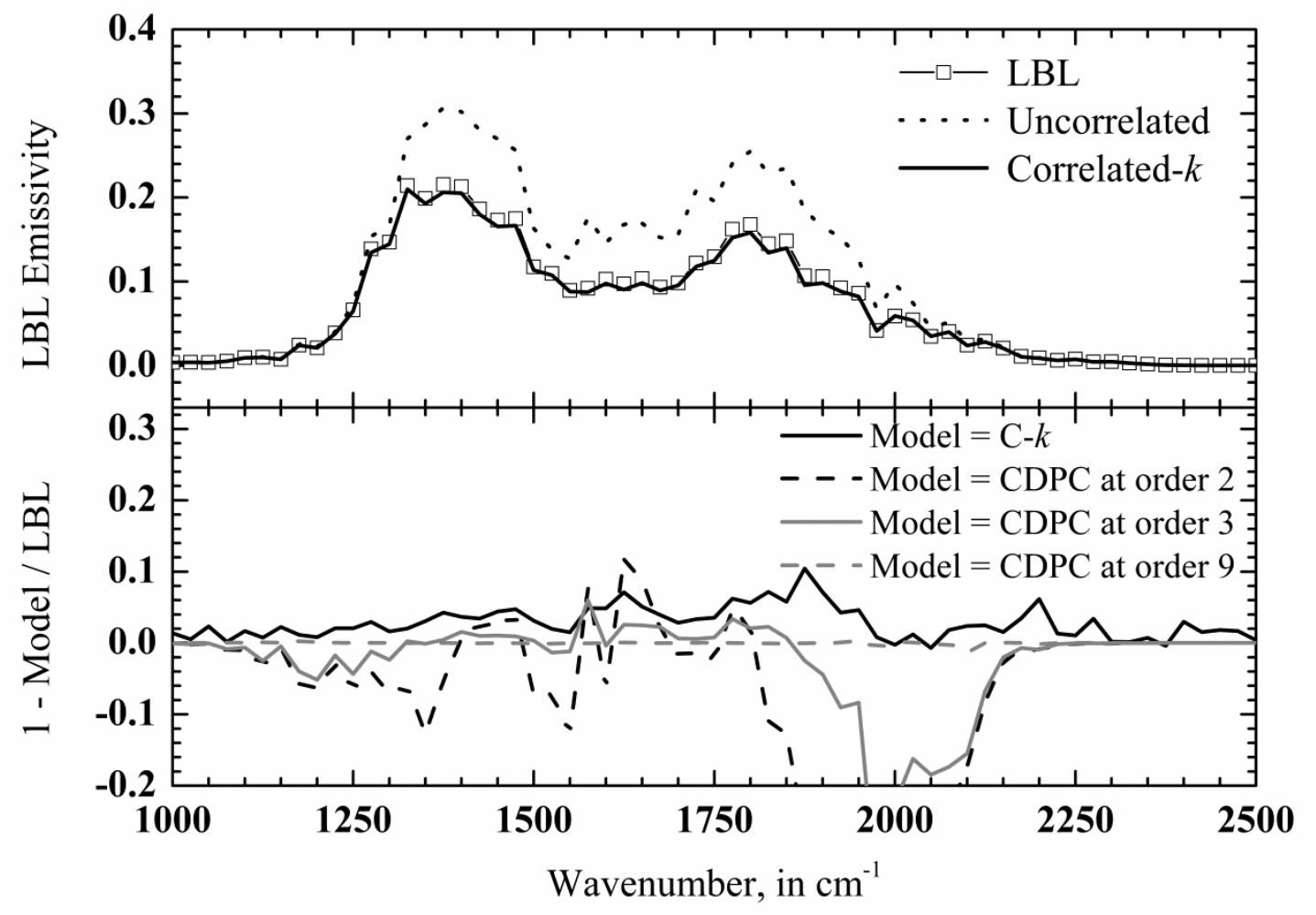

Figure 5. Narrow band emissivity for the $6.3 \mu \mathrm{m}$ band of $\mathrm{H}_{2} \mathrm{O}$ calculated by the LBL, uncorrelated, C- $k$ and CDPC (at orders 2, 3 and 9) models. $20 \% \mathrm{H}_{2} \mathrm{O}$ and $80 \% \mathrm{~N}_{2}$ at 1 atmosphere. The temperature (lengths) of the cold and hot paths are $300 \mathrm{~K}(50 \mathrm{~cm})$ and $500 \mathrm{~K}$ $(50 \mathrm{~cm})$ respectively. 


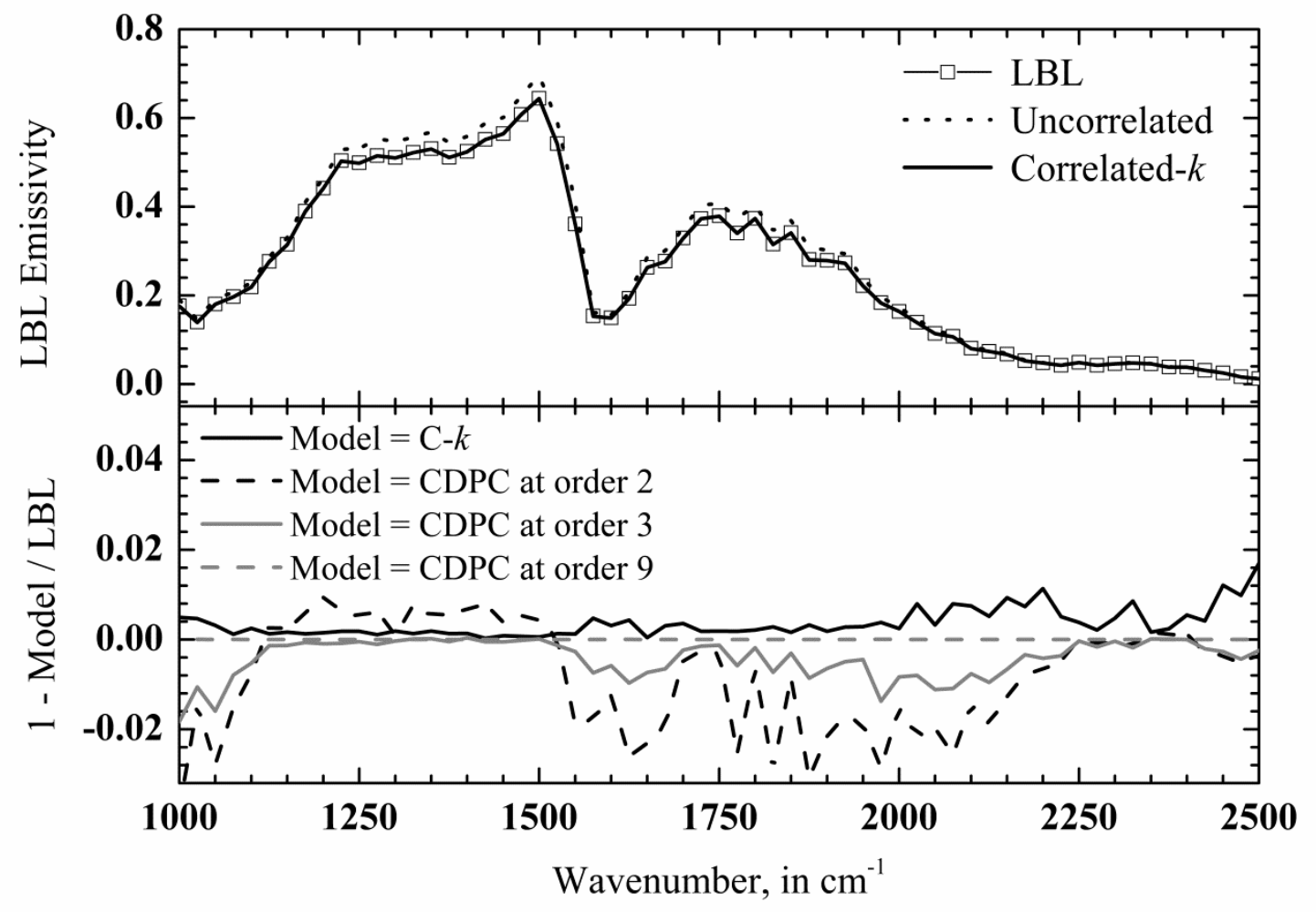

Figure 6. Narrow band emissivity for the $6.3 \mu \mathrm{m}$ band of $\mathrm{H}_{2} \mathrm{O}$ calculated by the LBL, uncorrelated, C- $k$ and CDPC (at orders 2, 3 and 9) models. $10 \% \mathrm{H}_{2} \mathrm{O}$ and $90 \% \mathrm{~N}_{2}$ in one layer; $20 \% \mathrm{H}_{2} \mathrm{O}-80 \% \mathrm{~N}_{2}$ in the other layer; both layers are at 1 atmosphere. The temperature (length) is the same in both columns: $2000 \mathrm{~K}(50 \mathrm{~cm})$. 


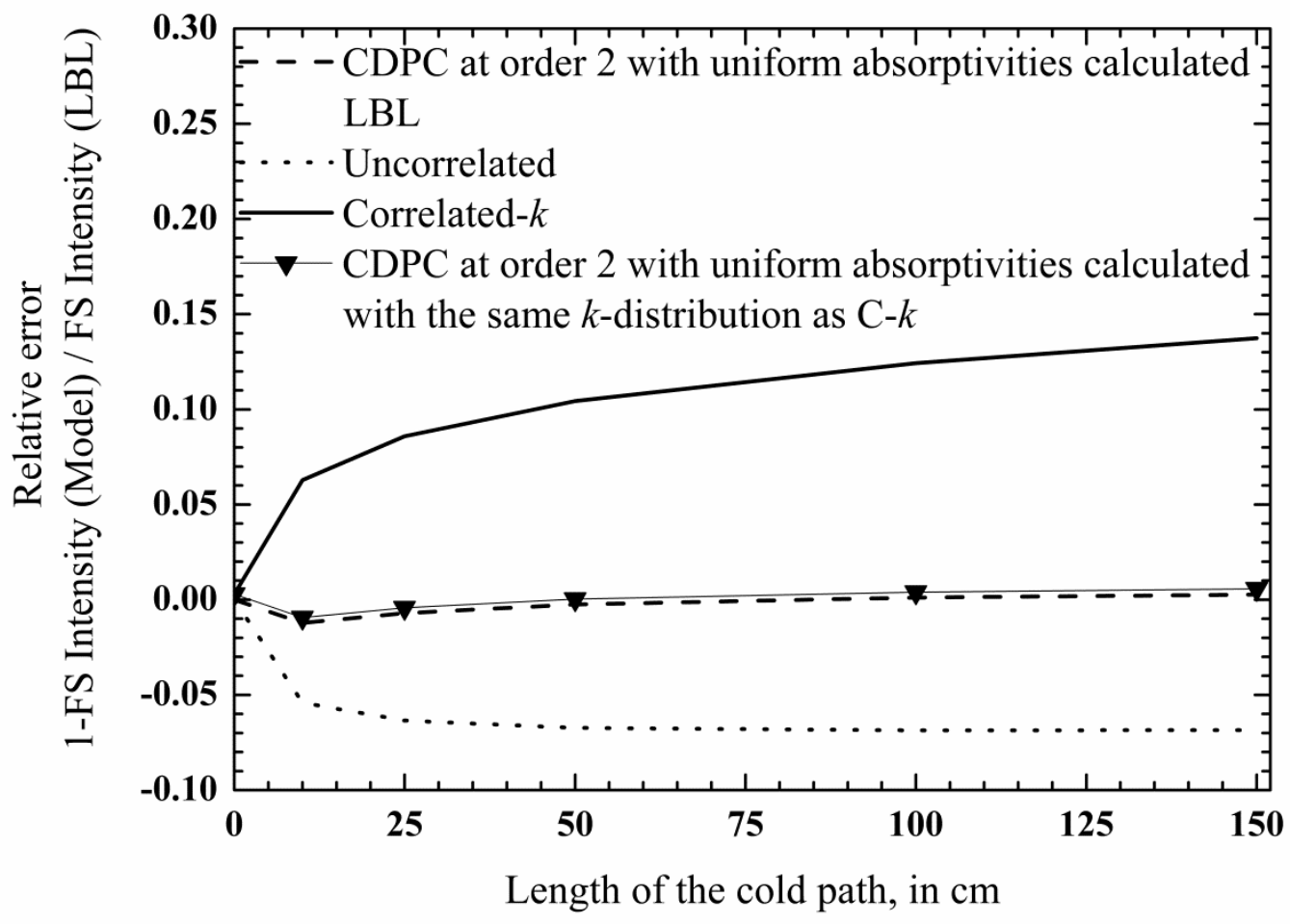

Figure 7. Relative errors on full spectrum intensities for various models as a function of the length of the cold path (see text for details). 


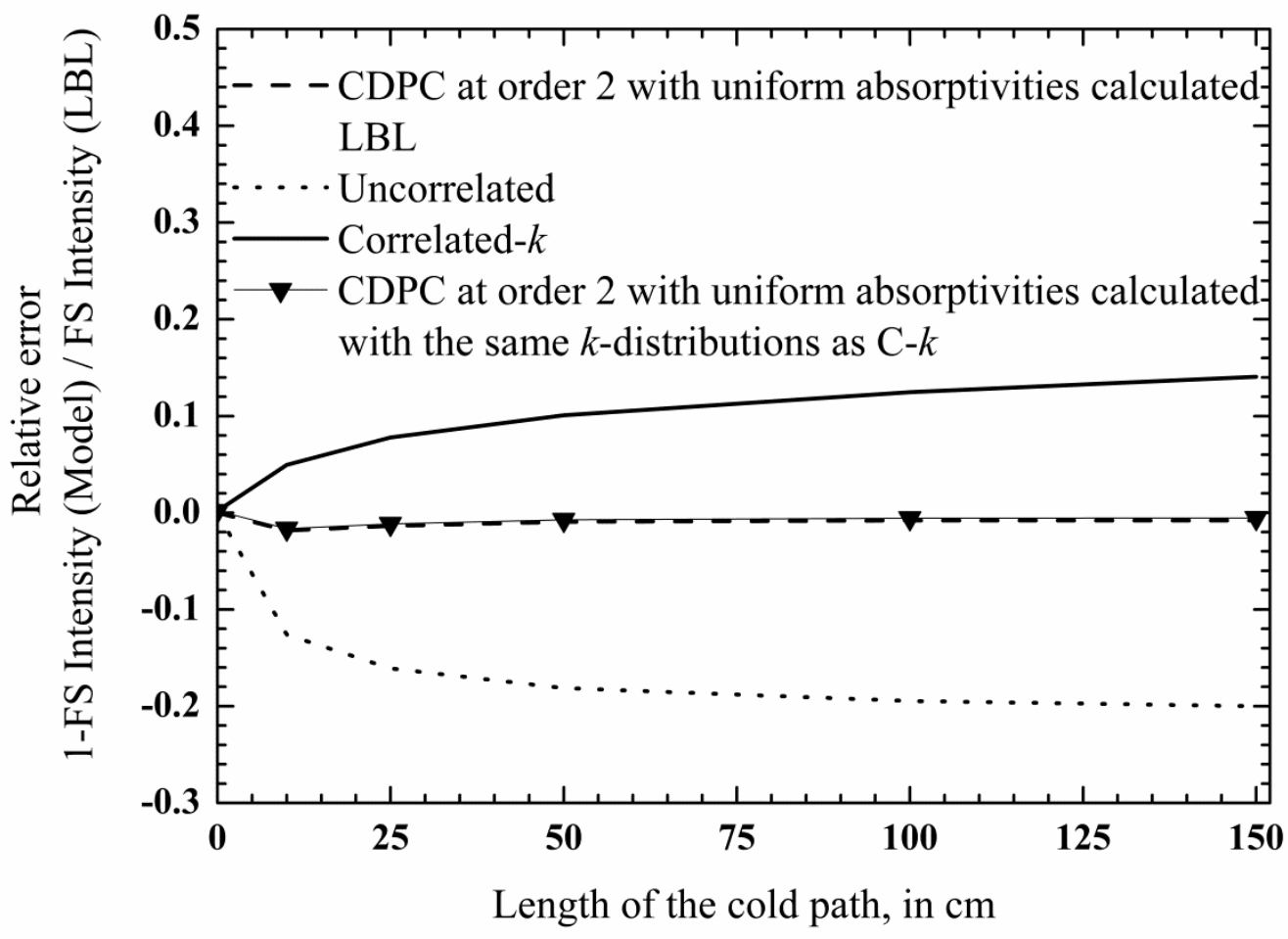

Figure 8. Relative errors on full spectrum intensities for various models as a function of the length of the cold path (see text for details). 


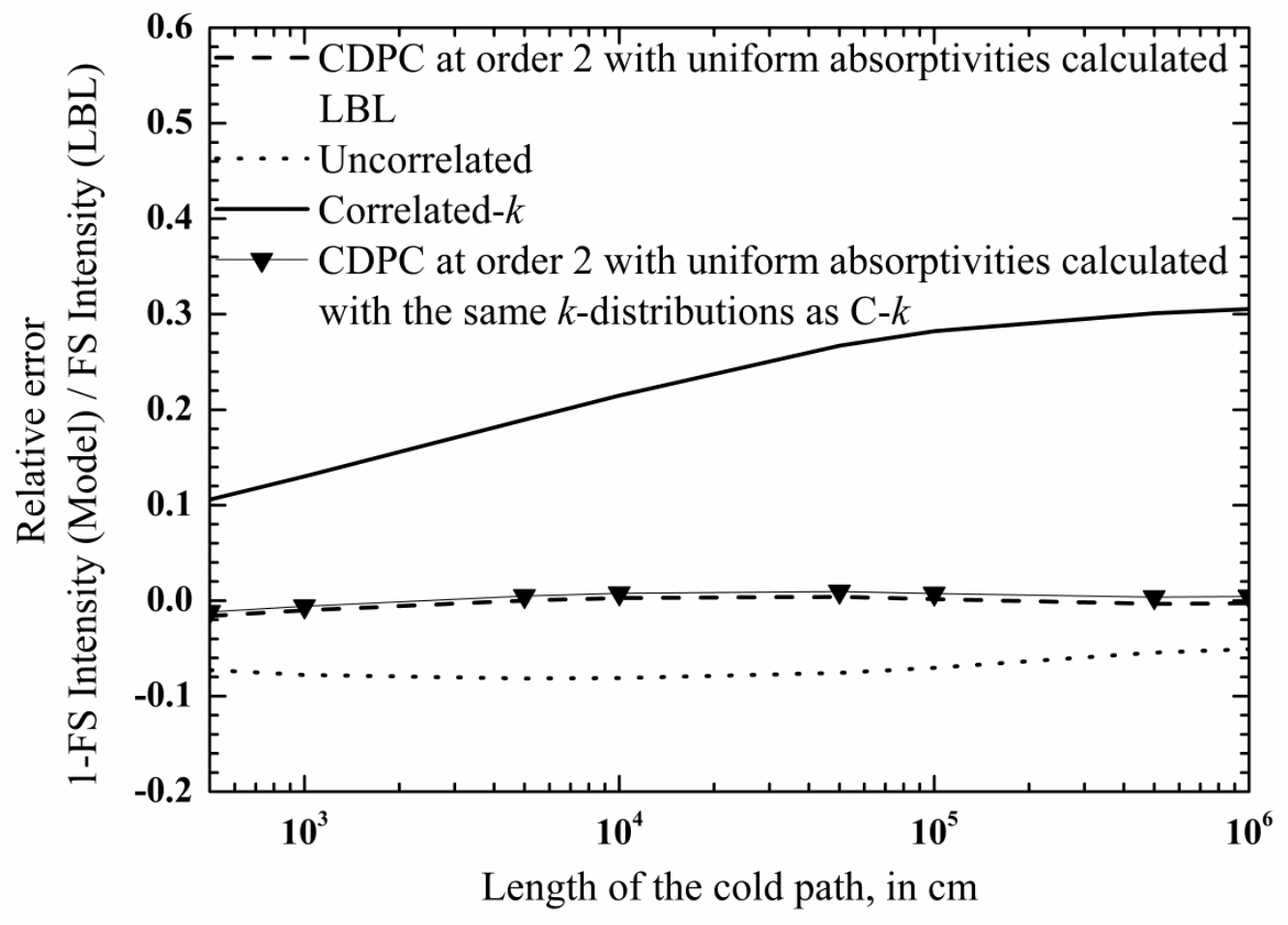

Figure 9. Relative errors on full spectrum intensities for various models as a function of the length of the cold path (see text for details). 


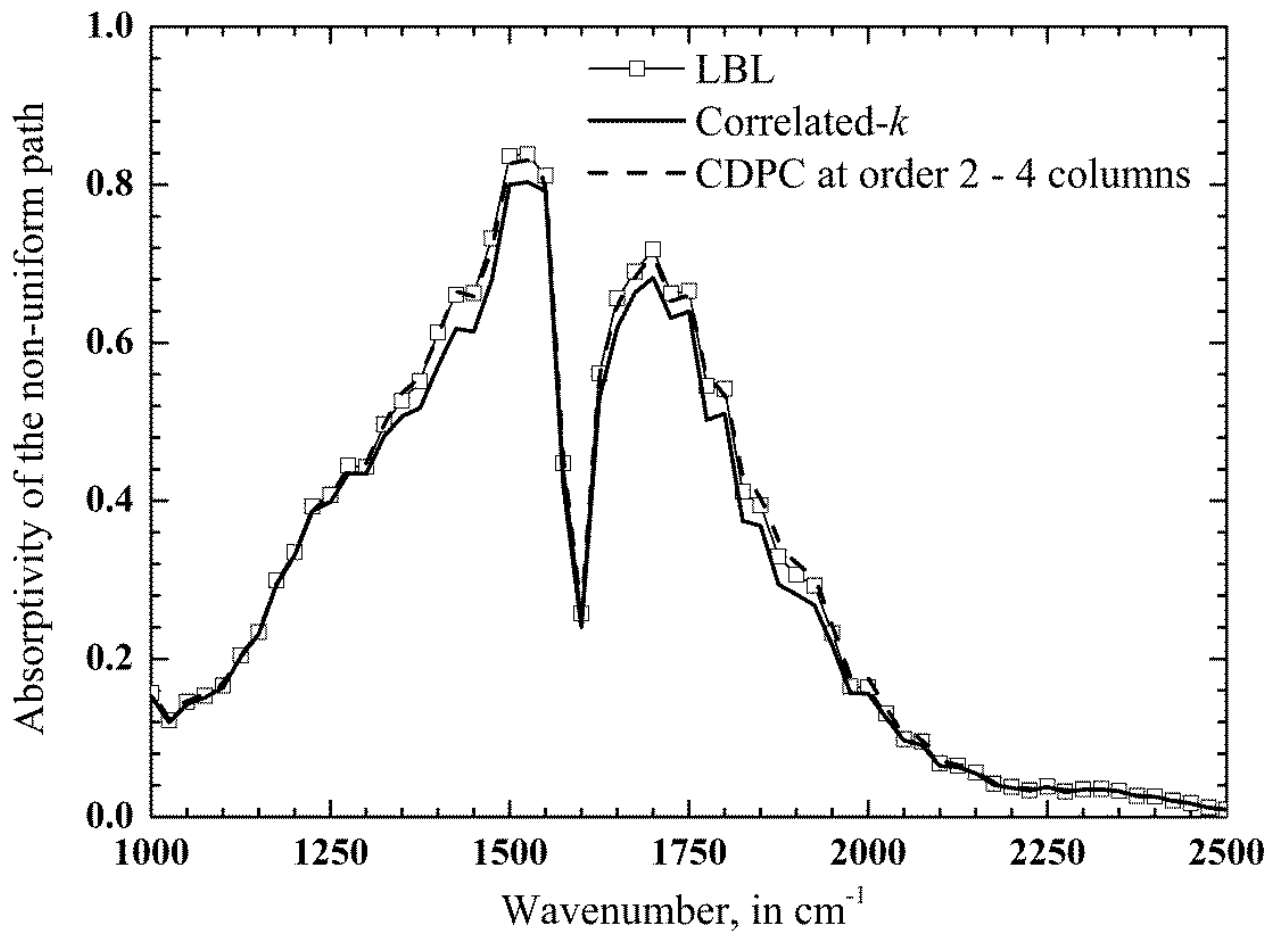

Figure 10. LBL, C- $k$ and CDPC (at order 2) models for 4 uniform columns (see text in Appendix for details). 


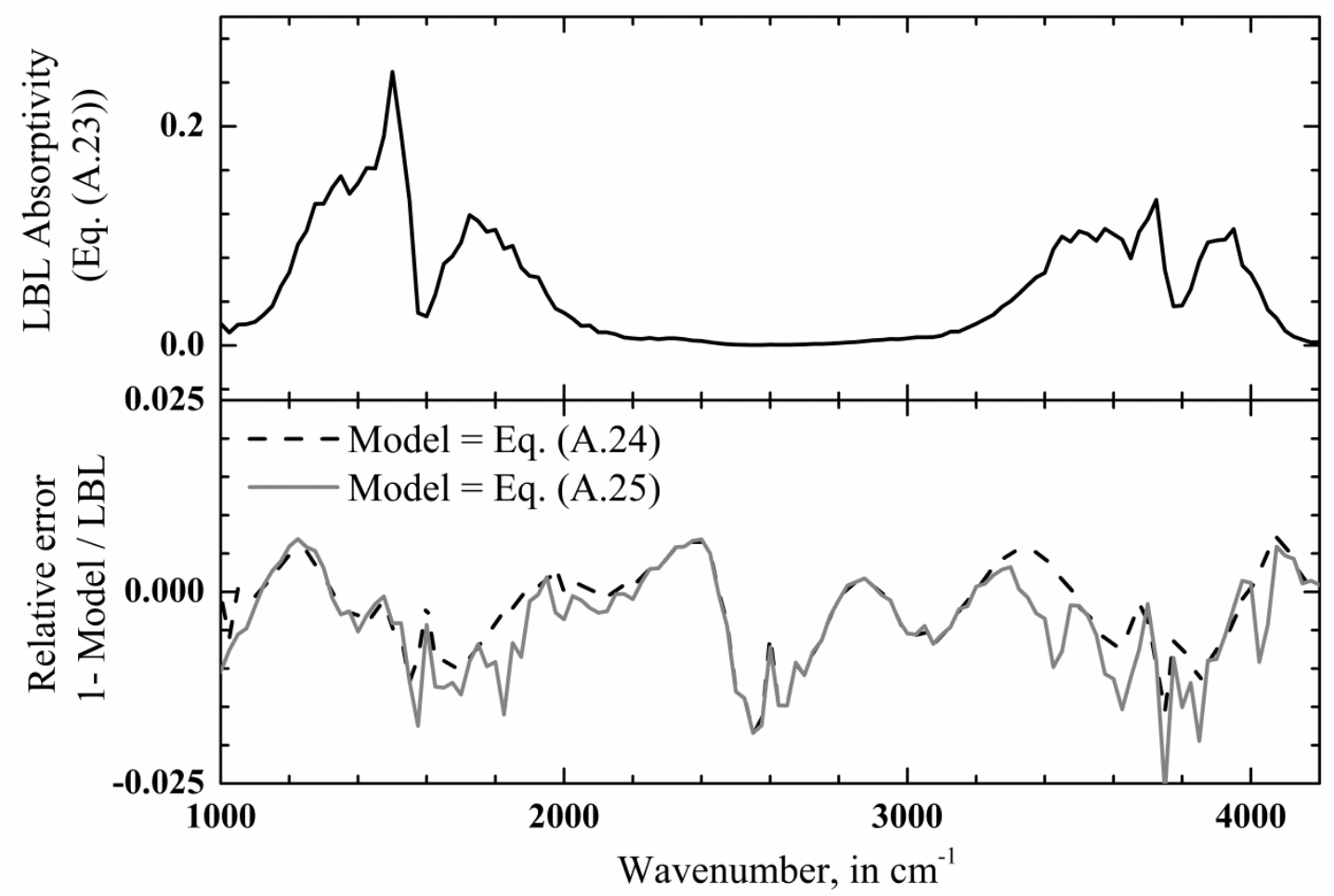

Figure 11. Accuracy of the CDPC model at order 2 when used with (linearly) interpolated spectra. 
TABLE

\begin{tabular}{|c|c|c|c|c|c|}
\hline Case & $\mathbf{1}-\mathbf{U n c} /$ & $\begin{array}{c}\mathbf{1}-\mathbf{C}-\boldsymbol{k} / \\
\mathbf{L B L}\end{array}$ & $\begin{array}{c}\mathbf{1}-\mathbf{C D P C} \text { at } \\
\text { order } \mathbf{2} \text { / LBL }\end{array}$ & $\begin{array}{c}\mathbf{1}-\mathbf{C D P C} \text { at } \\
\text { order 3 / LBL }\end{array}$ & $\begin{array}{c}\text { 1 - CDPC at } \\
\text { order 9 / LBL }\end{array}$ \\
\hline $\mathbf{C B L}$ & -6.1 & 11.0 & -0.3 & -0.1 & $<0.1^{*}$ \\
\hline $\mathbf{C 2}$ & -7.6 & 26.7 & 0.4 & -0.7 & $<0.1^{*}$ \\
\hline $\mathbf{C 3}$ & -19.9 & 6.3 & -2.6 & -0.3 & $<0.1^{*}$ \\
\hline $\mathbf{C 4}$ & -45.0 & 4.1 & -6.2 & -1.2 & $<0.1^{*}$ \\
\hline $\mathbf{C 5}$ & -6.8 & 0.2 & -0.5 & -0.3 & $<0.1^{*}$ \\
\hline
\end{tabular}

* $:$ «<0.1» means <0.1 in absolute value.

Table 1. Relative errors (in \%) for wide band calculations (see text for details) for Cases C15 . 


\section{APPENDIX. SOME USEFUL RESULTS}

The objective of this appendix is to provide evidences of some mathematical relationships used in the paper.

\section{Appendix 1. Calculation of some derivatives}

By taking the derivative of Eq. (7), we obtain:

$$
d \xi_{i}=\frac{\partial \alpha_{i}^{\Delta \eta}\left[L_{i}=l_{i}\left(\xi_{i}\right)\right]}{\partial L_{i}} d l_{i}\left(\xi_{i}\right)
$$

The joint derivative of function $C\left(\xi_{1}, \xi_{2}\right)$ can be calculated in a similar way as:

$$
\begin{aligned}
\frac{\partial^{2} C\left(\xi_{1}, \xi_{2}\right)}{\partial \xi_{1} \partial \xi_{2}} & =\frac{\partial^{2} \Gamma_{12}^{\Delta \eta}\left[l_{1}\left(\xi_{1}\right), l_{2}\left(\xi_{2}\right)\right]}{\partial \xi_{1} \partial \xi_{2}} \\
& =\left[\frac{1}{\Delta \eta} \int_{\Delta \eta} \kappa_{\eta, 1} \kappa_{\eta, 2} \exp \left(-\kappa_{\eta, 1} l_{1}\left(\xi_{1}\right)-\kappa_{\eta, 2} l_{2}\left(\xi_{2}\right)\right) d \eta\right] \frac{d l_{1}\left(\xi_{1}\right)}{d \xi_{1}} \frac{d l_{2}\left(\xi_{2}\right)}{d \xi_{2}} \\
& =\frac{\partial^{2} \Gamma_{12}^{\Delta \eta}\left[L_{1}=l_{1}\left(\xi_{1}\right), L_{2}=l_{2}\left(\xi_{2}\right)\right]}{\partial L_{1} \partial L_{2}} \frac{d l_{1}\left(\xi_{1}\right)}{d \xi_{1}} \frac{d l_{2}\left(\xi_{2}\right)}{d \xi_{2}}
\end{aligned}
$$

It can be rewritten, using the previous relationships:

$$
\frac{\partial^{2} C\left(\xi_{1}, \xi_{2}\right)}{\partial \xi_{1} \partial \xi_{2}}=\frac{\frac{\partial^{2} \Gamma_{12}^{\Delta \eta}\left[L_{1}=l_{1}\left(\xi_{1}\right), L_{2}=l_{2}\left(\xi_{2}\right)\right]}{\partial L_{1} \partial L_{2}}}{\frac{\partial \alpha_{1}^{\Delta \eta}\left[L_{1}=l_{1}\left(\xi_{1}\right)\right]}{\partial L_{1}} \frac{\partial \alpha_{2}^{\Delta \eta}\left[L_{2}=l_{2}\left(\xi_{2}\right)\right]}{\partial L_{2}}}
$$

Eq. (16) follows directly from the previous development.

Furthermore, as $\frac{\partial^{2} \Gamma_{12}^{\Delta \eta}\left[L_{1}=l_{1}\left(\xi_{1}\right), L_{2}=l_{2}\left(\xi_{2}\right)\right]}{\partial L_{1} \partial L_{2}} \geq 0$ (see above) as soon as assumption $H 2$ is true, it follows (from Eq. (3)) that $\frac{\partial^{2} C\left(\xi_{1}, \xi_{2}\right)}{\partial \xi_{1} \partial \xi_{2}} \geq 0$ for any set $\left(\xi_{1}, \xi_{2}\right) \in[0,1]^{2}$. This justifies that $C\left(\xi_{1}, \xi_{2}\right)$ has property $P 5$.

\section{Appendix 2. Some results on moments}

If we put the previous relationships into Eq. (16) and use Eq. (7) we obtain: 


$$
\begin{aligned}
\chi_{n m} & =\iint_{[0,1]^{2}} \frac{\partial^{2} C\left(\xi_{1}, \xi_{2}\right)}{\partial \xi_{1} \partial \xi_{2}} \xi_{1}^{n} \xi_{2}^{m} d \xi_{1} d \xi_{2} \\
& =\iint_{[0,1]^{2}} \frac{\partial^{2} \Gamma_{12}^{\Delta \eta}\left[L_{1}=l_{1}\left(\xi_{1}\right), L_{2}=l_{2}\left(\xi_{2}\right)\right]}{\partial L_{1} \partial L_{2}}\left\{\alpha_{1}^{\Delta \eta}\left[L_{1}=l_{1}\left(\xi_{1}\right)\right]\right\}^{n}\left\{\alpha_{2}^{\Delta \eta}\left[L_{2}=l_{2}\left(\xi_{2}\right)\right]\right\}^{m} d l_{1}\left(\xi_{1}\right) d l_{2}\left(\xi_{i}\right) \\
& =\int_{0}^{+\infty} \int_{0}^{+\infty} \frac{\partial^{2} \Gamma_{12}^{\Delta \eta}\left[L_{1}=l_{1}, L_{2}=l_{2}\right]}{\partial L_{1} \partial L_{2}}\left[\alpha_{1}^{\Delta \eta}\left(L_{1}=l_{1}\right)\right]^{n}\left[\alpha_{2}^{\Delta \eta}\left(L_{2}=l_{2}\right)\right]^{m} d l_{1} d l_{2}
\end{aligned}
$$

The evaluation of the previous integral can be done analytically at orders $(0,0),(0, m \geq 1)$ and $(n \geq 1,0)$ by permuting the integral signs.

Indeed, at order $n=0$ and $m=0$ :

$$
\begin{aligned}
& \chi_{00}=\int_{0}^{+\infty} \int_{0}^{+\infty} \frac{\partial^{2} \Gamma_{12}^{\Delta \eta}\left[L_{1}=l_{1}, L_{2}=l_{2}\right]}{\partial L_{1} \partial L_{2}} d l_{1} d l_{2}=\int_{0}^{+\infty} \int_{0}^{+\infty}\left[\frac{1}{\Delta \eta} \int_{\Delta \eta} \kappa_{\eta, 1} \kappa_{\eta, 2} \exp \left(-\kappa_{\eta, 1} l_{1}-\kappa_{\eta, 2} l_{2}\right) d \eta\right] d l_{1} d l_{2} \\
& =\int_{0}^{+\infty}\{\frac{1}{\Delta \eta} \int_{\Delta \eta} \kappa_{\eta, 1} \exp \left(-\kappa_{\eta, 1} l_{1}\right) \underbrace{\left[\int_{0}^{+\infty} \kappa_{\eta, 2} \exp \left(-\kappa_{\eta, 2} l_{2}\right) d l_{2}\right]}_{1} d \eta\} d l_{1} \\
& =\frac{1}{\Delta \eta} \int_{\Delta \eta} \underbrace{\left[\int_{0}^{+\infty} \kappa_{\eta, 1} \exp \left(-\kappa_{\eta, 1} l_{1}\right) d l_{1}\right]}_{1} d \eta \\
& =1
\end{aligned}
$$

Similarly, at orders $n \geq 1$ and $m=0$ :

$$
\begin{aligned}
& \chi_{n 0}=\int_{0}^{+\infty} \int_{0}^{+\infty} \frac{\partial^{2} \Gamma_{12}^{\Delta \eta}\left[L_{1}=l_{1}, L_{2}=l_{2}\right]}{\partial L_{1} \partial L_{2}}\left[\alpha_{1}^{\Delta \eta}\left(L_{1}=l_{1}\right)\right]^{n} d l_{1} d l_{2} \\
& =\int_{0}^{+\infty}\{\frac{1}{\Delta \eta} \int_{\Delta \eta} \kappa_{\eta, 1} \exp \left(-\kappa_{\eta, 1} l_{1}\right) \underbrace{\left[\int_{0}^{+\infty} \kappa_{\eta, 2} \exp \left(-\kappa_{\eta, 2} l_{2}\right) d l_{2}\right]}_{1} d \eta\}\left[\alpha_{1}^{\Delta \eta}\left(L_{1}=l_{1}\right)\right]^{n} d l_{1} \\
& =\int_{0}^{+\infty} \underbrace{\left\{\frac{1}{\Delta \eta} \int_{\Delta \eta} \kappa_{\eta, 1} \exp \left(-\kappa_{\eta, 1} l_{1}\right) d \eta\right\}}_{=\partial \alpha_{1}^{\Delta \eta}\left(L_{1}=l_{1}\right) / \partial L_{1}}\left[\alpha_{1}^{\Delta \eta}\left(L_{1}=l_{1}\right)\right]^{n} d l_{1} \\
& =\frac{1}{n+1} \int_{0}^{+\infty} \partial\left[\alpha_{1}^{\Delta \eta}\left(L_{1}=l_{1}\right)\right]^{n+1} / \partial L_{1} d l_{1} \\
& =\frac{1}{n+1}
\end{aligned}
$$


A symmetrical result can be obtained for $\chi_{0 m}$.

This shows that at order $N, 2 N-1$ coefficients over $N^{2}$ are known in the polynomial expansion. Accordingly, $(N-1)^{2}$ coefficients need to be evaluated to apply Eq. (14) at order $N$.

This means that for the CDPC model at order 2 (that requires 4 coefficients $\left.\chi_{n m}, n=0,1, m=0,1\right)$ only one coefficient is unknown and needs to be evaluated. This is one of the advantages of using CDPs in the present polynomial representation.

\section{Appendix 3. Some results on CDPs}

Cutteridge-Devyatov polynomials (CDPs) can be evaluated from the following set of equations (see Ref. [31]) for more details on how those polynomials are built):

$$
\Phi_{k}^{(N)}(u)=\sum_{n=0}^{N-1} \phi_{k n}^{(N)} u^{n+1}
$$

where:

$$
\phi_{k n}^{(N)}=(-1)^{k+n} \frac{(N+k) !(N+n) !}{k !(k+1) ! n !(n+1) !(N-k-1) !(N-n-1) !} \frac{k+1}{k+n+1}
$$

They are thus polynomials at order $N$.

At $\operatorname{order} N=1$, the previous relationship provides:

$$
\Phi_{0}^{(1)}(u)=u
$$

and, at order $N=2$ :

$$
\begin{gathered}
\Phi_{0}^{(2)}(u)=4 u-3 u^{2} \\
\Phi_{1}^{(2)}(u)=-6 u+6 u^{2}
\end{gathered}
$$

This provides, when used together with Eq. (14) the following approximations of function $C\left(\xi_{1}, \xi_{2}\right)$ :

- $\quad$ at $\operatorname{order} N=1(\mathrm{CDPC}$ model at order 1$)$ :

$$
C_{1}\left(\xi_{1}, \xi_{2}\right)=\chi_{00} \Phi_{0}^{(1)}\left(\xi_{1}\right) \Phi_{0}^{(1)}\left(\xi_{2}\right)=\xi_{1} \xi_{2}
$$

The CDPC model at order 1 thus provides the uncorrelated approximation Eq. (29). The copula associated with this expansion is known as the independence copula [20].

- $\quad$ at $\operatorname{order} N=2(\mathrm{CDPC}$ model at order 2): 


$$
\begin{aligned}
C_{2}\left(\xi_{1}, \xi_{2}\right) & =\chi_{00} \Phi_{0}^{(2)}\left(\xi_{1}\right) \Phi_{0}^{(2)}\left(\xi_{2}\right)+\chi_{01} \Phi_{0}^{(2)}\left(\xi_{1}\right) \Phi_{1}^{(2)}\left(\xi_{2}\right)+\chi_{10} \Phi_{1}^{(2)}\left(\xi_{1}\right) \Phi_{0}^{(2)}\left(\xi_{2}\right) \\
& +\chi_{11} \Phi_{1}^{(2)}\left(\xi_{1}\right) \Phi_{1}^{(2)}\left(\xi_{2}\right) \\
& =\Phi_{0}^{(2)}\left(\xi_{1}\right) \Phi_{0}^{(2)}\left(\xi_{2}\right)+\frac{1}{2} \Phi_{0}^{(2)}\left(\xi_{1}\right) \Phi_{1}^{(2)}\left(\xi_{2}\right)+\frac{1}{2} \Phi_{1}^{(2)}\left(\xi_{1}\right) \Phi_{0}^{(2)}\left(\xi_{2}\right) \\
& +\chi_{11} \Phi_{1}^{(2)}\left(\xi_{1}\right) \Phi_{1}^{(2)}\left(\xi_{2}\right)
\end{aligned}
$$

It can be simplified into (using the previous definitions of CDPs):

$$
C_{2}\left(\xi_{1}, \xi_{2}\right)=\xi_{1} \xi_{2}+\left(\chi_{11}-\frac{1}{4}\right) \Phi_{1}^{(2)}\left(\xi_{1}\right) \Phi_{1}^{(2)}\left(\xi_{2}\right)
$$

In the previous approximation, at order 2, only one unknown coefficient needs to be evaluated to allow the computation of $C_{2}$ : $\chi_{11}$. This follows directly from the results given in the previous section. When $\chi_{11}=1 / 4, C_{2}\left(\xi_{1}, \xi_{2}\right)$ and $C_{1}\left(\xi_{1}, \xi_{2}\right)$ are the same. This corresponds to the uncorrelated case.

It should be noticed that Eq. (A.14) does not ensure $C_{2}\left(\xi_{1}, \xi_{2}\right)$ to be a copula in a general frame. Indeed, properties $P 3$ and $P 4$ are obviously true. But the truthfulness of $P 5$ depends on the value of $\chi_{11}$.

However, if we put Eq. (A.11) into (A.14) we obtain:

$$
C_{2}\left(\xi_{1}, \xi_{2}\right)=\xi_{1} \xi_{2}\left[1+36\left(\chi_{11}-\frac{1}{4}\right)\left(1-\xi_{1}\right)\left(1-\xi_{2}\right)\right]
$$

This polynomial expansion corresponds to the so-called FGM (Farlie-Gumbel-Morgenstern) or EFGM (Eyraud-Farlie-Gumbel-Morgenstern) - [20] family of copulas if $-1 \leq 36\left(\chi_{11}-\frac{1}{4}\right) \leq 1$. This inequality was found to be true in cases $\mathrm{C} 1$ and $\mathrm{C} 2$, but not in cases C3-5. This means that in those situations, $C_{2}$ is not a FGM copula. However, as noticed in Table 1, this does not seem to have a strong impact on the quality of the results. Indeed, although C3-5 are the cases for which the CDPC model at order 2 performs the worst, its results over wide bands are as accurate as the usual C- $k$ model.

Finally, we can notice that for any integer order $N$, the polynomial approximation Eq. (14) can be written in the following form (similar to (A.15)):

$$
C_{N}\left(\xi_{1}, \xi_{2}\right)=\xi_{1} \xi_{2}\left[1+D_{N}\left(\xi_{1}, \xi_{2}\right)\right]
$$

Indeed, as noticed in Ref. [19], any polynomial $P: u \in[0,1] \rightarrow[a, b]$ at order $N$, where $[a, b]$ is some bounded interval on the real line, can be written in terms of CDPs as:

$$
P(u)=P(0)+\sum_{n=0}^{N-1} p_{n} \Phi_{n}^{(N)}(u)
$$


where:

$$
p_{n}=\int_{0}^{1} u^{n} \frac{d P(u)}{d u} d u
$$

The previous relationship can be used to write:

$$
u=\sum_{n=0}^{N-1}\left[\int_{0}^{1} u^{n} 1 d u\right] \Phi_{n}^{(N)}(u)=\sum_{n=0}^{N-1} \frac{1}{n+1} \Phi_{n}^{(N)}(u)
$$

Accordingly, we may write Eq. (14) as:

$$
\begin{aligned}
C\left(\xi_{1}, \xi_{2}\right) \approx & \sum_{n=0}^{N-1} \sum_{m=0}^{N-1} \chi_{n m} \Phi_{n}^{(N)}\left(\xi_{1}\right) \Phi_{m}^{(N)}\left(\xi_{2}\right) \\
= & \sum_{n=0}^{N-1} \sum_{m=0}^{N-1} \frac{1}{n+1} \frac{1}{m+1} \Phi_{n}^{(N)}\left(\xi_{1}\right) \Phi_{m}^{(N)}\left(\xi_{2}\right) \\
& +\sum_{n=0}^{N-1} \sum_{m=0}^{N-1}\left(\chi_{n m}-\frac{1}{n+1} \frac{1}{m+1}\right) \Phi_{n}^{(N)}\left(\xi_{1}\right) \Phi_{m}^{(N)}\left(\xi_{2}\right) \\
= & {\left[\sum_{n=0}^{N-1} \frac{1}{n+1} \Phi_{n}^{(N)}\left(\xi_{1}\right)\right]\left[\sum_{m=0}^{N-1} \frac{1}{m+1} \Phi_{m}^{(N)}\left(\xi_{2}\right)\right] } \\
& +\sum_{n=0}^{N-1} \sum_{m=0}^{N-1}\left(\chi_{n m}-\frac{1}{n+1} \frac{1}{m+1}\right) \Phi_{n}^{(N)}\left(\xi_{1}\right) \Phi_{m}^{(N)}\left(\xi_{2}\right)
\end{aligned}
$$

The first two terms at the RHS can be simplified, using Eq. (A.19), to yield:

$$
C\left(\xi_{1}, \xi_{2}\right) \approx \xi_{1} \xi_{2}\left[1+\sum_{n=1}^{N-1} \sum_{m=1}^{N-1}\left(\chi_{n m}-\frac{1}{n+1} \frac{1}{m+1}\right) \frac{\Phi_{n}^{(N)}\left(\xi_{1}\right)}{\xi_{1}} \frac{\Phi_{m}^{(N)}\left(\xi_{2}\right)}{\xi_{2}}\right]
$$

that is of the same form as Eq. (A.16) if we write:

$$
D_{N}\left(\xi_{1}, \xi_{2}\right)=\sum_{n=1}^{N-1} \sum_{m=1}^{N-1}\left(\chi_{n m}-\frac{1}{n+1} \frac{1}{m+1}\right) \frac{\Phi_{n}^{(N)}\left(\xi_{1}\right)}{\xi_{1}} \frac{\Phi_{m}^{(N)}\left(\xi_{2}\right)}{\xi_{2}}
$$

It can be noticed that in the previous equations, the sums start at $n=1$ and $m=1$. This is a direct consequence of Eq. (A.6). Furthermore, following the definition of the CDPs (Eq. (A.7)), the quantities $\frac{\Phi_{n}^{(N)}\left(\xi_{1}\right)}{\xi_{1}}$ and $\frac{\Phi_{m}^{(N)}\left(\xi_{2}\right)}{\xi_{2}}$ are polynomials at order $N-1$. The mathematical form (A.16) is interesting if the problem is formulated in terms of copulas [20]. It arises directly from the properties of the CDPs. 
Appendix 4. Some thought on the extension of the method to dimensions higher than 2.

The aim of this short section is to discuss the extension of the CDPC method to more than two uniform paths. Accordingly, other possible methods, such as those presented in Section 3.3., will not be discussed.

For this purpose we will consider two problems: 1/ does the method using CDPC at order 2 remain accurate if the number of uniform sub-paths is increased? 2/ how can we apply the technique to thermophysical states that are not inside the prescribed states, viz. those used to generate the moments - Eq. (28) -.

In order to study the first problem, we will consider a path subdivided into 4 uniform columns. The first one is at $2700 \mathrm{~K}$, the second one at $1900 \mathrm{~K}$, the third at $1100 \mathrm{~K}$ and the last one at $300 \mathrm{~K}$. All columns contain a mixture of $20 \% \mathrm{H}_{2} \mathrm{O}$ and $80 \% \mathrm{~N}_{2}$. The extension of the technique described in the paper to this new case requires (for the CDPC approach at order 2) $2^{4}=16$ coefficients, among which 5 are known (as a direct generalization of the results obtained for 2 columns). The narrow band averaged absorptivity of the non-uniform path is plotted in Figure 10 (all paths have the same length $L_{1}=L_{2}=L_{3}=L_{4}=10 \mathrm{~cm}$ ). Results for the $\mathrm{C}-k$ model are also given.

From this figure we can conclude that in this case with 4 uniform columns, the CDPC model at order 2 is more accurate than the C- $k$ model. The number of coefficients required for 4 columns remains acceptable (11). For $N$ columns, it is roughly $2^{N}$ (among which many are known).

The extension of the technique to any thermophysical state was studied by generating coefficients for the following couple of states $\left(20 \%\right.$ of $\mathrm{H}_{2} \mathrm{O}$ and $80 \%$ of $\mathrm{N}_{2}$ at $1400 \mathrm{~K}$ and $1600 \mathrm{~K})$. Then it was assumed that absorption spectra vary linearly with respect to temperature inside the interval $[1400 \mathrm{~K}, 1600 \mathrm{~K}]$. We have plotted in Figure 11 results for a calculation at $1500 \mathrm{~K}$ (this temperature is inside the previous interval). At the top, the absorptivity of a uniform path at $1500 \mathrm{~K}$ and length $L=10 \mathrm{~cm}$ calculated $\mathrm{LBL}$ is shown:

$$
\alpha_{L B L}^{\Delta \eta}(L)=\frac{1}{\Delta \eta} \int_{\Delta \eta}\left[1-\exp \left(-\kappa_{\eta}(T=1500 K) L\right)\right] d \eta
$$

At the bottom, two curves - relative errors when compared to the exact solution given by Eq. (A.23) - are depicted: the first one corresponds to a LBL calculation based on the assumption of linearity of absorption coefficients with respect to temperature over the interval $[1400 \mathrm{~K}, 1600 \mathrm{~K}]$ :

$$
\begin{aligned}
\alpha_{\text {lin }}^{\Delta \eta}(L)= & \frac{1}{\Delta \eta} \int_{\Delta \eta}\left[1-\exp \left(-\kappa_{\eta}(T=1400 K) \frac{(1600-T)}{1600-1400} L-\kappa_{\eta}(T=1600 K) \frac{(T-1400)}{1600-1400} L\right)\right] d \eta \\
& =\frac{1}{T=1500 K} \frac{1}{\Delta \eta} \int_{\Delta \eta}\left[1-\exp \left(-\kappa_{\eta}(T=1400 K) \frac{1}{2} L-\kappa_{\eta}(T=1600 K) \frac{1}{2} L\right)\right] d \eta
\end{aligned}
$$

The second one uses the following relationship, which arises directly from Eq. (A.24): 


$$
\begin{aligned}
\alpha_{C D P C}^{\Delta \eta}(L) & =\alpha_{T=1400 K}^{\Delta \eta}\left(\frac{1}{2} L\right)+\alpha_{T=1600 K}^{\Delta \eta}\left(\frac{1}{2} L\right) \\
& -\sum_{n=0}^{3} \sum_{m=0}^{3} c_{n m} P_{n}^{*}\left[\alpha_{T=1400 K}^{\Delta \eta}\left(\frac{1}{2} L\right)\right] P_{m}^{*}\left[\alpha_{T=1600 K}^{\Delta \eta}\left(\frac{1}{2} L\right)\right]
\end{aligned}
$$

It can be seen that most of the error - when applying Eq. (A.25) - arises from the linear assumption, since the two curves at the bottom are almost the same. This means that using an appropriate interpolation scheme (more sophisticated than the simple linear one proposed here) is likely to extend the present method to any possible thermophysical state with a higher accuracy - which is however acceptable - than obtained here.

The results provided in these last two figures suggest the following facts: 1/ the approach at order 2 can be extended to more than two uniform sub-paths. In the case considered here, it provides an accuracy higher than the usual C- $k$ model; $2 /$ it is possible to extend the technique to thermophysical states not included in the set used to estimate the coefficients. This requires an appropriate interpolation scheme between spectra from this set.

Future developments of the method will focus on: 1/ defining the number of thermophysical states required to map the full range of possible applications (which has an impact on the number of $\chi$ parameters); $2 /$ studying the possibility to propose an accurate interpolation scheme between the corresponding spectra. 